\title{
ON THE REGULARITY OF SOLUTIONS TO A NONLINEAR ULTRAPARABOLIC EQUATION ARISING IN MATHEMATICAL FINANCE*
}

\author{
Giovanna Citti, Andrea Pascucci, and Sergio Polidoro \\ Dipartimento di Matematica, Università di Bologna \\ Piazza di Porta S. Donato 5, 40127 Bologna, Italy
}

(Submitted by: G. Da Prato)

\begin{abstract}
We consider the following nonlinear degenerate parabolic equation which arises in some recent problems of mathematical finance:

$$
\partial_{x x} u+u \partial_{y} u-\partial_{t} u=f .
$$

Using a harmonic analysis technique on Lie groups, we prove that, if the solution $u$ satisfies condition $\partial_{x} u \neq 0$ in an open set $\Omega \subset \mathbb{R}^{3}$ and $f \in C^{\infty}(\Omega)$, then $u \in C^{\infty}(\Omega)$.
\end{abstract}

\section{INTRODUCTION}

In this paper we study the interior regularity properties of the solutions to the equation in the variables $z=(x, y, t) \in \mathbb{R}^{3}$

$$
L u \equiv \partial_{x x} u+u \partial_{y} u-\partial_{t} u=f
$$

satisfying the condition

$$
\partial_{x} u(z) \neq 0 \quad \forall z \in \Omega .
$$

This equation arises in mathematical finance, when studying agents' decisions under risk. The problem is the representation of agents' preferences over consumption processes. Epstein and Zin in [9] have proposed a utility functional which is the solution of a backward stochastic differential equation. Recently Antonelli, Barucci and Mancino [1] proposed a more sophisticated utility functional that takes into account some aspects of decision making, such as the agents' habit formation, which is described as a smoothed average of past consumption and expected utility. In that model the couple

*Investigation supported by the University of Bologna. Funds for selected research topics.

Accepted for publication March 2000.

AMS Subject Classifications: 35K57, 35K65, 35K70. 
of processes utility and habit is described by a system of backward-forward stochastic differential equations. The solution of such a system as a function of consumption and time satisfies the partial differential equation (1.1).

Several existence and uniqueness results are known for viscosity solutions of the Cauchy problem associated with equation (1.1), under different hypotheses on the initial data ([23], [10], [1]). However no regularity results are known. Here we are concerned with the regularity of the classical solutions of (1.1) (see Section 3 for the precise definition of classical solution). To this end, condition (1.2) is of crucial importance not only because it is suggested by the model, but also because equation (1.1) could have nonregular solutions if it is suppressed. For example any solution $u$ independent of the variable $x$ satisfies the Burgers equation

$$
u \partial_{y} u-\partial_{t} u=0
$$

which is of hyperbolic type. Our main result is the following.

Theorem 1.1. Let $\Omega$ be an open subset of $\mathbb{R}^{3}$ and let $f \in C^{\infty}(\Omega)$. If $u$ is a classical solution of equation (1.1) in $\Omega$ and satisfies condition (1.2), then $u \in C^{\infty}(\Omega)$.

The operator $L$ in (1.1) can be seen as a degenerate parabolic operator, and it can formally be represented as a sum of squares of nonlinear vector fields. Indeed if we set

$$
X=\partial_{x} \text { and } Y_{u}=u \partial_{y}-\partial_{t},
$$

then $L$ can be expressed as

$$
L u=X^{2} u+Y_{u} u .
$$

Condition (1.2) ensures that the vector fields $X, Y_{u}$ and their commutator $\left[X, Y_{u}\right]=\partial_{x} u \partial_{y}$ are linearly independent at every point. This fact suggests a link with the theory of Hörmander's operators. These operators can be written in the form

$$
H=\sum_{i=1}^{p} X_{i}^{2}+X_{0}
$$

where $X_{i}, i=0, \ldots, p(p \leq N)$, are linear, smooth vector fields in $\mathbb{R}^{N}$ whose generated Lie algebra has maximum rank at every point. It is well known that this last condition, called the Hörmander condition, yields that $H$ is hypoelliptic (see [14]). Under this condition there exists a fundamental solution $\Gamma$ of the equation (1.5) whose properties have been investigated by [14], [20], [21], [17]. In particular in these papers a control distance $d$ associated to the vector fields and their commutators has been introduced. Moreover 
estimates of $\Gamma$ and its derivatives are proved in terms of $d$. Things are particularly easy when the Lie algebra generated by $X_{1}, \ldots, X_{p}$ is nilpotent and stratified. In this case there exists a nonnegative integer $Q$, which is called the homogeneous dimension of the space, such that

$$
\Gamma(z, \zeta) \leq C d(z, \zeta)^{-Q+2}
$$

for every $z, \zeta \in \Omega$. Hence a theory of the regularity similar to the classical one has been developed for this type of operator. If we denote by $C_{d}^{k, \alpha}$ the class of functions with derivatives of order $k$ Hölder continuous with respect to the control distance $d$, then some a priori estimates formally analogous to the classical Schauder ones hold for the solutions of the equation $\mathrm{Hu}=f$ (see [13], [11], [20]). Obviously, if $\bar{u}$ is a fixed function satisfying (1.2), then the linear operator

$$
L_{\bar{u}} u=X^{2} u+Y_{\bar{u}} u
$$

is formally represented as in (1.5), and the associated classes of Höldercontinuous functions will be denoted by $C_{\bar{u}}^{k, \alpha}$. Then we have the following result (see, for example, [20]):

Theorem RS1. Let the coefficient $\bar{u}$ of $L_{\bar{u}}$ be of class $C^{\infty}(\Omega)$, and let $f \in C_{\bar{u}}^{k-2, \alpha}(\Omega), k \in \mathbb{N}, 0<\alpha<1$. If $u$ is a solution of $L_{\bar{u}} u=f$, then $u$ is of class $C_{\bar{u}}^{k, \alpha}(\Omega)$.

This result is optimal if the coefficient $\bar{u}$ is of class $C^{\infty}$, and it can be easily extended with the same technique to less-regular vector fields. In this case, the following holds.

Theorem RS2. Assume that $\bar{u} \in C_{\bar{u}}^{k+1, \alpha}(\Omega)$ and $f \in C_{\bar{u}}^{k-2, \alpha}(\Omega)$. If $u$ is a solution of $L_{\bar{u}} u=f$, then $u$ is of class $C_{\bar{u}}^{k, \alpha}(\Omega)$.

We stress that this result can not be applied in our nonlinear situation, since the vector fields in (1.3) have only the regularity of the solution; then Theorem RS2 does not provide any gain of regularity. Hence we adapt to this framework a technique introduced by one of the authors in [6] and extended in [7] for studying the regularity of the solutions of another nonlinear operator. Here we are able to prove the following.

Theorem 1.2. Assume that $\Omega \subseteq \mathbb{R}^{3}$ is an open set, $\bar{u} \in C_{\bar{u}}^{k-1, \alpha}(\Omega)$ satisfies (1.2), and $f \in C_{\bar{u}}^{k-2, \alpha}(\Omega)$, for any $\alpha \in(0,1)$. If $u$ is a classical solution of $L_{\bar{u}} u=f$ in $\Omega$, then $u \in C_{\bar{u}}^{k, \alpha}(\Omega)$, for any $\alpha \in(0,1)$.

This result easily yields Theorem 1.1. The proof of Theorem 1.2 is established in two steps: a freezing method and a regularization procedure. 
1.1. Overview of the freezing method. The freezing method is a wellknown technique, classically used to study the regularity of solutions to linear parabolic operators of the form

$$
\sum_{i, j=1}^{N} a_{i j} \partial_{x_{i}} \partial_{x_{j}}-\partial_{t}
$$

where $z=(x, t)$ denotes the point in $\mathbb{R}^{N} \times \mathbb{R}$. In this case, the associated frozen operator is simply obtained by evaluating the coefficients at a fixed point $z_{0}$ :

$$
\sum_{i, j=1}^{N} a_{i j}\left(z_{0}\right) \partial_{x_{i}} \partial_{x_{j}}-\partial_{t}
$$

This new operator is, up to a linear change of coordinates, the heat operator, and its fundamental solution can be considered as a parametrix of the fundamental solution of the operator in (1.8). An argument much more complicated was used to prove the existence of a fundamental solution for Hörmander-type operators (1.5). Indeed the properties of the operator rely on the vector fields $X_{1}, \ldots, X_{p}$ and their commutators. If $X_{i}$ are represented by

$$
X_{i}=\sum_{j=1}^{N} a_{i j} \partial_{x_{j}} \quad i=1, \ldots, p,
$$

then the constant-coefficient operators

$$
\widetilde{X}_{i, z_{0}}=\sum_{j=1}^{N} a_{i j}\left(z_{0}\right) \partial_{x_{j}} \quad i=1, \ldots, p
$$

commute, and the generated Lie algebra is $\mathbb{R}^{p}$ with $p \leq N$. Hence, in general, the operator $\sum_{i=1}^{p} \widetilde{X}_{i, z_{0}}^{2}-\widetilde{X}_{0, z_{0}}$ is not hypoelliptic, and it has not a fundamental solution. Folland and Stein first pointed out that the model operators in this case are operators of the form (1.5) such that the Lie algebra generated by $X_{1} \cdots X_{p}$ is nilpotent and stratified. Later on Rothschild and Stein introduced an abstract and very general version of the freezing method. The choice of the frozen vector fields $X_{i, z_{0}}$ was made in such a way that their generated Lie algebra is nilpotent and stratified, and, at low orders, it has the same structure as $\operatorname{Lie}\left(X_{1} \cdots X_{p}\right)$. With this choice of vector fields, the operator $\sum_{i=1}^{p} X_{i, z_{0}}^{2}-X_{0, z_{0}}$ is hypoelliptic and nilpotent, and its fundamental solution $\Gamma_{z_{0}}$ is a parametrix for the fundamental solution of (1.5). After the existence of the fundamental solution of (1.5) was established, a wide class of Hörmander operators has been studied with the same argument as (1.8): the 
operators of the form $\sum_{i, j=1}^{p} a_{i j} X_{i} X_{j}+X_{0}$, where $a_{i, j}$ are not even continuous but the vector fields $X_{i}$ are of class $C^{\infty}$ and satisfy the Hörmander condition for hypoellipticity. Here, for every point $z_{0}$, it is convenient to consider as frozen operator $\sum_{i, j=1}^{p} a_{i j}\left(z_{0}\right) X_{i} X_{j}+X_{0}$, that is an operator with $C^{\infty}$ coefficients. In the same spirit as the results known for elliptic operators, by using the known properties of these operators, many sophisticated results have been obtained under very weak hypotheses on $a_{i j}$ (see, for example, [15], [19], [3], [2]). See also [16] where the regularity properties of this kind of operator have been investigated by a different approach.

The few known results about nonlinear operators refer to operators whose nonlinearity depends on $C^{\infty}$ vector fields (see, for example, [25], [4], [24], $[26])$.

Things are different when the vector fields themselves are not smooth, since that operators can not always be considered as simple perturbations of known linear operators. A first regularity result for solutions of a linear equation with continuous vector fields is due to Franchi and Lanconelli [12]. In a more recent study one of the authors introduced a simplification of the freezing method of Rothschild and Stein, for a second-order partial differential operator, based on the notion of "intrinsic" Taylor expansion of the coefficients [6].

In this paper we use a technique similar to the one in [6]. We consider the linearized operator $L_{\bar{u}}$ in (1.7) defined in an open subset $\Omega$ of $\mathbb{R}^{3}$. Assuming (1.2), we have that $X, Y_{\bar{u}},\left[X, Y_{\bar{u}}\right]$ are linearly independent at every point. We observe that the simplest nilpotent Lie algebra with two generators and of dimension 3 is the Heisenberg algebra. Then, for every point $z_{0} \in \Omega$, we associate with $X$ and $Y_{\bar{u}}$ two frozen vector fields $X$ and $Y_{z_{0}}$ of class $C^{\infty}$ and whose generated Lie algebra is the Heisenberg one. This choice ensures that the frozen operator $L_{z_{0}}=X^{2}+Y_{z_{0}}$ is a nilpotent Hörmander-type operator. In particular $L_{z_{0}}$ has a fundamental solution $\Gamma_{z_{0}}$ and an associated control distance $d_{z_{0}}$. Unfortunately the distance $d_{z_{0}}$ is not equivalent to the distance $d_{\bar{u}}$ associated with $L_{\bar{u}}$, nor are equivalent two distances $d_{z_{0}}$ and $d_{z_{1}}$ associated with different points $z_{0}$ and $z_{1}$. Since the qualitative behaviour of the solution strictly depends on the control distance, we have to study in detail the properties of these distances. This is done in Section 2 where we also study the properties of the Hölder classes related to these control distances.

1.2. Overview of the regularization procedure. In order to introduce our regularity procedure we consider a solution $u$ in $\Omega$ of the linearized equation $L_{\bar{u}} u=f$. Fixing $z_{0} \in \Omega$, we represent the function $u$ in terms of 
the fundamental solution $\Gamma_{z_{0}}$ of the frozen operator $L_{z_{0}}$ :

$$
\begin{aligned}
u(z) & =\int \Gamma_{z_{0}}(z, \zeta) L_{z_{0}} u(\zeta) d \zeta \\
& =\int \Gamma_{z_{0}}(z, \zeta) f(\zeta) d \zeta+\int \Gamma_{z_{0}}(z, \zeta) K_{z_{0}}(\zeta) d \zeta,
\end{aligned}
$$

where $K_{z_{0}}$ is a kernel with the behaviour $K_{z_{0}}(\zeta) \sim d_{z_{0}}^{q}\left(z_{0}, \zeta\right)$, and the exponent $q$ depends on the regularity of $\bar{u}$. In their classical paper [20], Rothschild and Stein choose $z_{0}=z$ in the representation formula (1.9). Therefore the kernel which appears in the second term of (1.9) becomes $\Gamma_{z}(z, \zeta) K_{z}(\zeta)$, and it is less singular then $\Gamma_{z_{0}}$. Hence it is possible to perform higher-order derivatives with respect to $z$ and to estimate them. On the other hand, Chiarenza, Frasca and Longo [5] noticed for the first time that, even in the parabolic case, it seems to be convenient, when dealing with nonregular coefficients, to keep $z$ different from $z_{0}$ as long as it is possible. Using their technique in our situation, we can differentiate twice $u$ with respect to $z$, and we obtain

$$
D^{2} u(z)=\int D^{2} \Gamma_{z_{0}}(z, \zeta) f(\zeta) d \zeta+\int D^{2} \Gamma_{z_{0}}(z, \zeta) K_{z_{0}}(\zeta) d \zeta
$$

Then, we evaluate the second order derivative of $u$ at $z_{0}$ :

$$
D^{2} u\left(z_{0}\right)=\int D^{2} \Gamma_{z_{0}}\left(z_{0}, \zeta\right) f(\zeta) d \zeta+\int D^{2} \Gamma_{z_{0}}\left(z_{0}, \zeta\right) K_{z_{0}}(\zeta) d \zeta .
$$

In this way we compute the second derivative of $u$ without differentiating the coefficient of $\Gamma_{z_{0}}$. The same idea has been used in [7] also for higherorder derivatives, and here we further extend it. Obviously, we can not repeat the preceding arguments for the third derivatives since, for $z \neq z_{0}$, the kernel $D^{3} \Gamma_{z_{0}}(z, \zeta) K_{z_{0}}(\zeta)$ is not locally integrable. Nevertheless, a rather delicate argument, based on the use of some high-order difference quotients (see Section 3), yields

$$
D^{3} u\left(z_{0}\right)=\int D \Gamma_{z_{0}}\left(z_{0}, \zeta\right) D^{2} f(\zeta) d \zeta+\int D^{3} \Gamma_{z_{0}}\left(z_{0}, \zeta\right) K_{z_{0}}(\zeta) d \zeta .
$$

In this way, we obtain some regularity results for the solutions even though the coefficients of the vector fields and of the fundamental solution of the frozen operator are not regular.

\section{FreEZING METHOD}

In this section we describe the freezing method for the linear equation

$$
L=X^{2}+Y_{\bar{u}},
$$


where $X=\partial_{x}, Y_{\bar{u}}=\bar{u} \partial_{y}-\partial_{t}$ and $\bar{u}$ is a given function satisfying (1.2). In Subsection 2.1, we study the relation between the distances $d_{\bar{u}}$, associated with the vector fields $X$ and $Y_{\bar{u}}$, and the distance $d_{z_{0}}$ associated with the vector fields $X$ and $Y_{z_{0}}$. In Subsection 2.2, we define classes $C_{\bar{u}}^{k, \alpha}$ of Hölder-continuous functions with respect to the distance $d_{\bar{u}}$, and we prove the existence of a polynomial expansion of Taylor type for functions of class $C_{\bar{u}}^{k, \alpha}$. Finally, in Subsection 2.3, we study the properties of the fundamental solution of the frozen operator.

2.1. Heisenberg group and distances. Here we recall some properties of the Heisenberg algebra and we establish some relations between the control distances corresponding to the linear and to the frozen operators.

The Heisenberg algebra is a Lie algebra with two generators, and nilpotent of step two. The simplest representation of the Heisenberg vector fields is

$$
X_{H}=\partial_{\theta_{1}}-\frac{\theta_{2}}{2} \partial_{\theta_{3}} \text { and } Y_{H}=\partial_{\theta_{2}}+\frac{\theta_{1}}{2} \partial_{\theta_{3}} .
$$

Clearly $\left[X_{H}, Y_{H}\right]=\partial_{\theta_{3}}$ and all the other commutators vanish. The associated Lie group $\mathbb{H}_{1}$ is then $\mathbb{R}^{3}$, endowed with the following composition law:

$$
\theta \oplus \theta^{\prime}=\left(\theta_{1}+\theta_{1}^{\prime}, \theta_{2}+\theta_{2}^{\prime}, \theta_{3}+\theta_{3}^{\prime}+\frac{1}{2}\left(\theta_{1} \theta_{2}^{\prime}-\theta_{2} \theta_{1}^{\prime}\right)\right) .
$$

A natural dilations group on $\mathbb{H}_{1}$ is defined by

$$
\delta_{\lambda}^{H}(\theta)=\left(\lambda \theta_{1}, \lambda^{2} \theta_{2}, \lambda^{3} \theta_{3}\right), \quad \lambda>0 .
$$

Since the Jacobian $J \delta_{\lambda}^{H}=\lambda^{6}$, the homogeneous dimension of $\mathbb{H}_{1}$ with respect to $\left(\delta_{\lambda}^{H}\right)_{\lambda>0}$ is the exponent $Q=6$. A norm homogeneous with respect to this dilations group is given by $\|\theta\|_{H}=\left|\theta_{1}\right|+\left|\theta_{2}\right|^{\frac{1}{2}}+\left|\theta_{3}\right|^{\frac{1}{3}}$. The associated distance is obviously defined by $d_{H}\left(\theta^{\prime}, \theta\right)=\left\|\theta^{-1} \oplus \theta^{\prime}\right\|_{H}$. Clearly $X_{H}$ and $Y_{H}$ are respectively $\delta_{\lambda}^{H}$-homogeneous of degree one and two; that is,

$$
X_{H}\left(u \circ \delta_{\lambda}^{H}\right)=\lambda\left(X_{H} u\right) \circ \delta_{\lambda}^{H}, \quad Y_{H}\left(u \circ \delta_{\lambda}^{H}\right)=\lambda^{2}\left(Y_{H} u\right) \circ \delta_{\lambda}^{H} .
$$

Thus, the second-order differential operator $L_{H}=X_{H}^{2}+Y_{H}$ has a fundamental solution $\Gamma_{H}$ which is invariant with respect to the left $\oplus$-translations and $\delta_{\lambda}^{H}$-homogeneous of degree $-Q+2$.

We next introduce the canonical coordinates corresponding to the linear operator $L_{\bar{u}}$. If $D$ is a Lipschitz-continuous vector field, and $[0,1]$ is contained in the domain of the local solution to the Cauchy problem

$$
\gamma^{\prime}(s)=D(\gamma(s)), \quad \gamma(0)=z,
$$


we let $\exp (D)(z)=\gamma(1)$ and call an exponential map the application $D \mapsto$ $\exp (D)(z)$. Since $\gamma(s)=\exp (s D)(z)$, then $\exp (D)(z)$ is defined for $D$ sufficiently small. If $D_{1}, D_{2}, D_{3}$ are Lipschitz-continuous vector fields, linearly independent at every point, then the map

$$
F_{z}: \theta \mapsto \exp (\theta \cdot D)(z)=\exp \left(\theta_{1} D_{1}+\theta_{2} D_{2}+\theta_{3} D_{3}\right)(z)
$$

is a diffeomorphism of a neighborhood of the origin of $\mathbb{R}^{3}$ to a neighborhood $U_{z}$ of $z$. Its inverse function $\theta_{D, z}=F_{z}^{-1}$ defines the canonical change of variable associated with the vector field $D$, and center $z$. When $D_{1}, D_{2}, D_{3} \in$ $C^{1}$, by the properties of the solutions of the Cauchy problem, the Jacobian matrix $J F_{z}$ of the function $F_{z}$ depends continuously on $z$. Then, by the local invertibility theorem, the open set $U_{z}$ continuously depends on $z$.

By our assumptions, $X, Y_{\bar{u}}$ and $\left[X, Y_{\bar{u}}\right]=\bar{u}_{x} \partial_{y}$ are linearly independent at every point, but $\bar{u}_{x}$ is not Lipschitz continuous, so we cannot define $\exp \left(\theta \cdot\left(X, Y_{\bar{u}},\left[X, Y_{\bar{u}}\right]\right)\right)$. We instead consider

$$
\nabla_{\bar{u}}=\left(X, Y_{\bar{u}}, \partial_{y}\right)
$$

and denote by $\theta_{\bar{u}, z_{0}}$ the associated canonical change of coordinates, defined on $U_{z_{0}} \subseteq \Omega$. This function allows us to introduce a topological structure in a neighborhood of $z_{0}$, naturally associated with the vector fields $X$ and $Y_{\bar{u}}$. Indeed, by the continuity of $U_{z}$, there exists $r=r\left(z_{0}\right)>0$ such that the Euclidean ball $B\left(z_{0}, r\right)$ satisfies

$$
B\left(z_{0}, r\right) \subseteq U_{z}, \quad \forall z \in B\left(z_{0}, r\right) .
$$

Thus, if $z, \zeta \in B\left(z_{0}, r\right)$, then $\zeta \in U_{z}, \theta_{\bar{u}, z}(\zeta)$ is defined, and we can set

$$
d_{\bar{u}}(z, \zeta)=\left\|\theta_{\bar{u}, z}(\zeta)\right\|_{H} .
$$

More explicitly, we have

$$
\theta_{\bar{u}, z}(\zeta)=\left(\xi-x,-(\tau-t), \eta-y+(\tau-t) \int_{0}^{1} \bar{u}(\gamma(s)) d s\right),
$$

and

$$
d_{\bar{u}}(z, \zeta)=|\xi-x|+|\tau-t|^{\frac{1}{2}}+\left|\eta-y+(\tau-t) \int_{0}^{1} \bar{u}(\gamma(s)) d s\right|^{\frac{1}{3}},
$$

where $\gamma(s)=\exp \left(s \theta_{\bar{u}, z}(\zeta) \cdot \nabla_{\bar{u}}\right)(z)$ with $s \in[0,1]$.

Remark 2.1. Let $z_{0} \in \Omega$ and $z \in U_{z_{0}}$. An integral curve of $\nabla_{\bar{u}}$, connecting $z$ and $z_{0}$, is $\gamma(s)=\exp \left(s \theta_{\bar{u}, z_{0}}(z) \cdot \nabla_{\bar{u}}\right)\left(z_{0}\right)$ with $s \in[0,1]$. Then, for every $s \in[0,1]$, we have

$$
d_{\bar{u}}\left(\gamma(s), z_{0}\right)=\left\|s \theta_{\bar{u}, z_{0}}(z)\right\|_{H} \leq\left\|\theta_{\bar{u}, z_{0}}(z)\right\|_{H}=d_{\bar{u}}\left(z_{0}, z\right) .
$$


Since $\theta_{\bar{u}, z}(\zeta)$ is only a local diffeomorphism, it does not introduce a group structure on $\mathbb{R}^{3}$. In order to overcome this problem, we define a new vector field in the following way: for every fixed $z_{0} \in \Omega$, we define the frozen operator of $Y_{\bar{u}}$ as follows: $Y_{z_{0}}=Y_{\bar{u}\left(z_{0}\right)+\bar{u}_{x}\left(z_{0}\right)\left(x-x_{0}\right)}$, where we have denoted, as usual, $u_{x}\left(z_{0}\right)=\partial_{x} u\left(z_{0}\right)$. Obviously $X, Y_{z_{0}}$ and $\left[X, Y_{z_{0}}\right]=\bar{u}_{x}\left(z_{0}\right) \partial_{y}$ are of class $C^{\infty}$ and all the commutators of higher order are null. Now we set $\nabla_{z_{0}}=\left(X, Y_{z_{0}},\left[X, Y_{z_{0}}\right]\right)$ and $L_{z_{0}}=X^{2}+Y_{z_{0}}$. The map $\theta \mapsto \exp \left(\theta \cdot \nabla_{z_{0}}\right)(z)$ is a global diffeomorphism. We denote by $\theta_{z}^{\left(z_{0}\right)}$ the canonical coordinates associated with $\nabla_{z_{0}}$ and of center $z$. As a consequence of the CampbellHausdorff formula we have

$$
\begin{aligned}
& X\left(u \circ \theta_{z_{0}}^{\left(z_{0}\right)}\right)=\left(X_{H} u\right) \circ \theta_{z_{0}}^{\left(z_{0}\right)}, \quad Y_{z_{0}}\left(u \circ \theta_{z_{0}}^{\left(z_{0}\right)}\right)=\left(Y_{H} u\right) \circ \theta_{z_{0}}^{\left(z_{0}\right)}, \\
& \bar{u}_{x}\left(z_{0}\right) \partial_{y}\left(u \circ \theta_{z_{0}}^{\left(z_{0}\right)}\right)=\left(\partial_{\theta_{3}} u\right) \circ \theta_{z_{0}}^{\left(z_{0}\right)} .
\end{aligned}
$$

Then, as a direct consequence, we have $L_{z_{0}}\left(u \circ \theta_{z_{0}}^{\left(z_{0}\right)}\right)=\left(L_{H} u\right) \circ \theta_{z_{0}}^{\left(z_{0}\right)}$. The diffeomorphism $\theta_{z_{0}}^{\left(z_{0}\right)}$ naturally induces a Lie group structure with dilations on $\mathbb{R}^{3}$. Indeed, we define the composition law

$$
z \circ \zeta=\left(\theta_{z_{0}}^{\left(z_{0}\right)}\right)^{-1}\left(\theta_{z_{0}}^{\left(z_{0}\right)}(z) \oplus \theta_{z_{0}}^{\left(z_{0}\right)}(\zeta)\right)
$$

the dilations

$$
\delta_{\lambda}^{\left(z_{0}\right)}(z)=\left(\theta_{z_{0}}^{\left(z_{0}\right)}\right)^{-1}\left(\delta_{\lambda}^{H}\left(\theta_{z_{0}}^{\left(z_{0}\right)}(z)\right)\right), \quad \lambda>0
$$

and the function $d_{z_{0}}$ defined by

$$
d_{z_{0}}(z, \zeta)=\left\|\left(\theta_{z_{0}}^{\left(z_{0}\right)}(z)\right)^{-1} \oplus \theta_{z_{0}}^{\left(z_{0}\right)}(\zeta)\right\|_{H}
$$

which is a quasi-distance, in the sense that there exists a positive constant $\widetilde{C}=\widetilde{C}\left(z_{0}\right)$ such that

$$
d_{z_{0}}\left(z_{0}, \zeta\right) \leq \widetilde{C}\left(d_{z_{0}}\left(z_{0}, \eta\right)+d_{z_{0}}(\eta, \zeta)\right), \quad z, \eta, \zeta \in \mathbb{R}^{3} .
$$

Then $G_{z_{0}}=\left(\mathbb{R}^{3}, \circ\right)$ is the Lie group associated with the Lie algebra $\mathcal{L}_{z_{0}}=$ $\operatorname{Lie}\left(X, Y_{z_{0}}\right)$, generated by $X$ and $Y_{z_{0}}$, and it is isomorphic to $\mathbb{H}_{1}$. The quasidistance we have introduced can be represented as

$$
d_{z_{0}}(z, \zeta)=\left\|\theta_{z}^{\left(z_{0}\right)}(\zeta)\right\|_{H}, \quad z, \zeta \in \mathbb{R}^{3}
$$

and, more explicitly,

$$
\begin{aligned}
& \theta_{z}^{\left(z_{0}\right)}(\zeta)= \\
& \left(\xi-x,-(\tau-t), \frac{1}{\bar{u}_{x}\left(z_{0}\right)}\left(\eta-y+(\tau-t)\left(\bar{u}\left(z_{0}\right)+\bar{u}_{x}\left(z_{0}\right) \frac{\xi+x-2 x_{0}}{2}\right)\right)\right), \\
& d_{z_{0}}(z, \zeta)=
\end{aligned}
$$


$|\xi-x|+|\tau-t|^{\frac{1}{2}}+\left|\frac{1}{\bar{u}_{x}\left(z_{0}\right)}\left(\eta-y+(\tau-t)\left(\bar{u}\left(z_{0}\right)+\bar{u}_{x}\left(z_{0}\right) \frac{\xi+x-2 x_{0}}{2}\right)\right)\right|^{\frac{1}{3}}$.

We next describe some relations between $d_{z_{0}}$ and $d_{\bar{u}}$.

Remark 2.2. In the sequel, we shall also use the distance defined by

$$
\widetilde{d}_{z_{0}}(z, \zeta)=\left\|\widetilde{\theta}_{z}^{\left(z_{0}\right)}(\zeta)\right\|_{H}, \quad \widetilde{\theta}_{z}^{\left(z_{0}\right)}(\zeta)=\left(\xi-x,-(\tau-t), \eta-y+(\tau-t) \bar{u}\left(z_{0}\right)\right) .
$$

This distance is equivalent to $d_{z_{0}}$, in the sense that there exists a positive constant $C$, which depends only on $\bar{u}_{x}\left(z_{0}\right)$, such that

$$
\frac{1}{C} \widetilde{d}_{z_{0}}\left(z_{0}, \zeta\right) \leq d_{z_{0}}\left(z_{0}, \zeta\right) \leq C \widetilde{d}_{z_{0}}\left(z_{0}, \zeta\right), \quad \forall \zeta \in \mathbb{R}^{3} .
$$

Here and in the sequel, $C$ will denote a constant which will not always be the same. The proof of the above statement relies only on the following elementary inequality,

$$
(a b)^{\frac{1}{3}} \leq \frac{1}{3} a+\frac{2}{3} b^{\frac{1}{2}}, \quad \forall a, b>0,
$$

and on the explicit expression of $d_{z_{0}}$ provided in (2.9).

Lemma 2.3. Let $U_{z_{0}} \subset \subset \Omega$ be a neighborhood of $z_{0}$ such that $\left(\theta_{1}, \theta_{2}, \theta_{3}\right) \equiv$ $\theta_{\bar{u}, z_{0}}(\zeta)$ is defined for every $\zeta \in U_{z_{0}}$. If $\left(\widetilde{\theta}_{1}, \widetilde{\theta}_{2}, \widetilde{\theta}_{3}\right) \equiv \widetilde{\theta}_{z_{0}}^{\left(z_{0}\right)}(\zeta)$ is defined as in (2.10), then we have

$$
\left|\theta_{3}-\widetilde{\theta}_{3}\right| \leq C\left|\tau-t_{0}\right| d_{\bar{u}}\left(z_{0}, \zeta\right), \quad \forall \zeta \in U_{z_{0}} .
$$

Proof. Since $\bar{u}$ is a locally Lipschitz-continuous function (in the Euclidean sense), we get

$$
\left|\bar{u}(\gamma(s))-\bar{u}\left(z_{0}\right)\right| \leq C_{1}\left|\gamma(s)-z_{0}\right| \leq C_{2} d_{\bar{u}}\left(z_{0}, \gamma(s)\right),
$$

where $\gamma(s)=\exp \left(s \theta_{\bar{u}, z_{0}}(\zeta) \cdot \nabla_{\bar{u}}\right)\left(z_{0}\right)$, for $s \in[0,1]$, and $C_{1}, C_{2}$ depend only on $U_{z_{0}}$. Thus the assertion follows from expressions (2.3) and (2.10):

$$
\left|\theta_{3}-\widetilde{\theta}_{3}\right| \leq\left|\tau-t_{0}\right| \int_{0}^{1}\left|\bar{u}(\gamma(s))-\bar{u}\left(z_{0}\right)\right| d s \leq C_{2}\left|\tau-t_{0}\right| \int_{0}^{1} d_{\bar{u}}\left(z_{0}, \gamma(s)\right) d s
$$

(by Remark 2.1) $\leq C_{2}\left|\tau-t_{0}\right| d_{\bar{u}}\left(z_{0}, \zeta\right)$.

Proposition 2.4. For every $z_{1} \in \Omega$, there exists a compact neighborhood $K \subseteq \Omega$ of $z_{1}$, and a positive constant $C=C(K)$, such that

i) $C^{-1} d_{z_{0}}\left(z_{0}, \zeta\right) \leq d_{\bar{u}}\left(z_{0}, \zeta\right) \leq C d_{z_{0}}\left(z_{0}, \zeta\right)$,

ii) $d_{z_{0}}\left(z_{0}, z\right) \leq C\left(d_{z_{0}}\left(z_{0}, \zeta\right)+d_{\zeta}(\zeta, z)\right)$,

iii) $d_{\bar{u}}\left(z_{0}, z\right) \leq C\left(d_{\bar{u}}\left(z_{0}, \zeta\right)+d_{\bar{u}}(\zeta, z)\right)$, for every $z, z_{0}, \zeta \in K$. 
Proof. We first remark that there exists $r=r\left(z_{1}\right)>0$ such that

$$
K \equiv B\left(z_{1}, r\right) \subseteq U_{z_{0}} \cap U_{z} \cap U_{\zeta}
$$

(i) If $\theta_{\bar{u}, z_{0}}(\zeta)$ and $\widetilde{\theta}_{z_{0}}^{\left(z_{0}\right)}(\zeta)$ are the functions defined in (2.3) and (2.10), they have the first two components in common. Then, using Lemma 2.3, we get

$$
\begin{aligned}
\widetilde{d}_{z_{0}}\left(z_{0}, \zeta\right) & =\left\|\widetilde{\theta}_{z_{0}}^{\left(z_{0}\right)}(\zeta)\right\|_{H} \leq\left\|\theta_{\bar{u}, z_{0}}(\zeta)\right\|_{H}+\left|\theta_{3}-\widetilde{\theta}_{3}\right|^{\frac{1}{3}} \\
& \leq d_{\bar{u}}\left(z_{0}, \zeta\right)+C\left|\tau-t_{0}\right|^{\frac{2}{3}} d_{\bar{u}}\left(z_{0}, \zeta\right)^{\frac{1}{3}} \leq C_{1} d_{\bar{u}}\left(z_{0}, \zeta\right)
\end{aligned}
$$

On the other hand, again from Lemma 2.3 and (2.11), we get

$$
\left|\theta_{3}-\tilde{\theta}_{3}\right| \leq C\left|\tau-t_{0}\right| d_{\bar{u}}\left(z_{0}, \zeta\right) \leq C\left(\frac{2}{3}\left(\frac{\left|\tau-t_{0}\right|}{\delta}\right)^{\frac{3}{2}}+\frac{1}{3}\left(\delta d_{\bar{u}}\left(z_{0}, \zeta\right)\right)^{3}\right)
$$

for any positive $\delta$. Therefore

$$
\begin{aligned}
d_{\bar{u}}\left(z_{0}, \zeta\right) & \leq \widetilde{d}_{z_{0}}\left(z_{0}, \zeta\right)+\left|\theta_{3}-\widetilde{\theta}_{3}\right|^{\frac{1}{3}} \\
& \leq\left(\delta^{-\frac{1}{2}}\left(\frac{2 C}{3}\right)^{\frac{1}{3}}+1\right) \widetilde{d}_{z_{0}}\left(z_{0}, \zeta\right)+\delta\left(\frac{C}{3}\right)^{\frac{1}{3}} d_{\bar{u}}\left(z_{0}, \zeta\right)
\end{aligned}
$$

By choosing a suitably small $\delta>0$ and by Remark 2.2, we get the thesis.

(ii) We observe that

$$
\begin{aligned}
\mid y-y_{0} & +\left.\bar{u}\left(z_{0}\right)\left(t-t_{0}\right)\right|^{\frac{1}{3}} \leq|y-\eta+\bar{u}(\zeta)(t-\tau)|^{\frac{1}{3}} \\
& +\left|\eta-y_{0}+\bar{u}\left(z_{0}\right)\left(\tau-t_{0}\right)\right|^{\frac{1}{3}}+\left|(t-\tau)\left(\bar{u}\left(z_{0}\right)-\bar{u}(\zeta)\right)\right|^{\frac{1}{3}} \\
& \leq \widetilde{d}_{\zeta}(\zeta, z)+\widetilde{d}_{z_{0}}\left(z_{0}, \zeta\right)+\left(|t-\tau| d_{\bar{u}}\left(z_{0}, \zeta\right)\right)^{\frac{1}{3}},
\end{aligned}
$$

since $\bar{u}$ is Lipschitz continuous. The last term can be estimated by (2.11) and (i), as follows:

$$
\left|(t-\tau) d_{\bar{u}}\left(z_{0}, \zeta\right)\right|^{\frac{1}{3}} \leq \frac{2}{3}|t-\tau|^{\frac{1}{2}}+C_{1} d_{\bar{u}}\left(z_{0}, \zeta\right) \leq \frac{2}{3} \widetilde{d}_{\zeta}(\zeta, z)+C \widetilde{d}_{z_{0}}\left(z_{0}, \zeta\right)
$$

By the definition of $\widetilde{d}_{z_{0}}\left(z_{0}, z\right)$, this last inequality and Remark 2.2 yield the assertion.

(iii) It is a direct consequence of (i) and (ii).

Remark 2.5. Since we are proving a local result, from now on we shall always work in a compact set $K \subseteq \Omega$, satisfying the assumptions of Proposition 2.4 . 


\subsection{Hölder-continuous functions and Taylor polynomials.}

Definition 2.6. Let $z_{0} \in \Omega, 0<\alpha<1$ and let $D$ be a locally Lipschitz continuous vector field on $\Omega$. We say that $u \in C_{D}^{\alpha}\left(z_{0}\right)$ if there exists a positive constant $C$ such that

$$
\left|u\left(\exp (h D)\left(z_{0}\right)\right)-u\left(z_{0}\right)\right| \leq C|h|^{\alpha},
$$

for every suitably small $h$. We say that $u \in C_{D}^{\alpha}(\Omega)$, if (2.13) holds uniformly on compact subsets of $\Omega$.

Definition 2.7. Let $z_{0} \in \Omega$ and $D$ be a Lipschitz-continuous vector field in $\Omega$. We say that there exists the Lie derivative of $u$ with respect to $D$ in $z_{0}$, if the following limit exists:

$$
D u\left(z_{0}\right) \equiv \lim _{h \rightarrow 0} \frac{u\left(\exp (h D)\left(z_{0}\right)\right)-u\left(z_{0}\right)}{h} .
$$

We denote by $C_{\bar{u}}^{0}(\Omega)$ (or $C_{\bar{u}}(\Omega)$ ) the set of continuous functions in $\Omega$. If $u \in C_{\bar{u}}(\Omega)$, and there exists $X u \in C_{\bar{u}}(\Omega)$, we say that $u \in C_{\bar{u}}^{1}(\Omega)$. If $k \geq 2$, $X u \in C_{\bar{u}}^{k-1}(\Omega)$ and $Y_{\bar{u}} u \in C_{\bar{u}}^{k-2}(\Omega)$, then we say that $u \in C_{\bar{u}}^{k}(\Omega)$.

Remark 2.8. We remark that if $u \in C^{1}(\Omega)$ and $D$ can be expressed as $D=d_{1} \partial_{x}+d_{2} \partial_{y}+d_{3} \partial_{t}$, with $d_{1}, d_{2}, d_{3}$ Lipschitz-continuous functions, then there exists the Lie derivative of $u$ and it can be expressed as

$$
D u\left(z_{0}\right)=d_{1} \partial_{x} u\left(z_{0}\right)+d_{2} \partial_{y} u\left(z_{0}\right)+d_{3} \partial_{t} u\left(z_{0}\right), \quad z_{0} \in \Omega .
$$

We next define the spaces of Hölder-continuous functions related to the linear operator $L_{\bar{u}}$.

Definition 2.9. Let $\bar{u}$ be a $C^{1}$ function satisfying (1.2), and let $0<\alpha<1$.

We say that $u \in C_{\bar{u}}^{\alpha}(\Omega)$ if $u \in C_{X}^{\alpha}(\Omega)$ and $u \in C_{Y_{\bar{u}}}^{\frac{\alpha}{2}}(\Omega)$.

We say that $u \in C_{\bar{u}}^{1, \alpha}(\Omega)$ if $X u \in C_{\bar{u}}^{\alpha}(\Omega)$ and $u \in C_{Y_{\bar{u}}}^{\frac{1+\alpha}{2}}(\Omega)$.

We say that $u \in C_{\bar{u}}^{2, \alpha}(\Omega)$ if $X u \in C_{\bar{u}}^{1, \alpha}(\Omega)$ and $Y_{\bar{u}} u \in C_{\bar{u}}^{\alpha}(\Omega)$.

Let $k \geq 3$ and suppose that $\bar{u} \in C_{\bar{u}}^{k-2, \alpha}(\Omega)$. We say that $u \in C_{\bar{u}}^{k, \alpha}(\Omega)$ if $X u \in C_{\bar{u}}^{k-1, \alpha}(\Omega)$ and $Y_{\bar{u}} u \in C_{\bar{u}}^{k-2, \alpha}(\Omega)$.

For the sake of simplicity, in the sequel, when we write $u \in C_{\bar{u}}^{k, \alpha}(\Omega)$, for $k \geq 3$, we will assume implicitly that $\bar{u} \in C_{\bar{u}}^{k-2, \alpha}(\Omega)$.

Remark 2.10. For every fixed $z \in \Omega$, we agree to work only in a compact neighborhood $K$ of $z_{1}$ satisfying the assumptions of Proposition 2.4. Thus $d_{\bar{u}}\left(z_{0}, z\right)$ is defined for every $z_{0}, z \in K$. We will see that, as a simple consequence of Theorem 2.16, that the class $C_{\bar{u}}^{\alpha}(\Omega)$ is defined in such a way that, 
for every $u \in C_{\bar{u}}^{\alpha}(\Omega)$,

$$
\left|u(z)-u\left(z_{0}\right)\right| \leq C d_{\bar{u}}\left(z_{0}, z\right)^{\alpha}, \quad \forall z, z_{0} \in K .
$$

Remark 2.11. In the sequel we will use the following simple result: if $0<\alpha<1$ and $k \geq 1$, then $C_{\bar{u}}^{k, \alpha}(\Omega) \subseteq C_{\bar{u}}^{k-1, \beta}(\Omega), \forall \beta \in(0,1)$.

We next prove some regularity results in the direction $\partial_{y}$ for a function belonging to the spaces $C_{\bar{u}}^{k, \alpha}(\Omega)$.

Proposition 2.12. Let $\bar{u}$ be a $C^{1}$-function satisfying (1.2) and let $0<\alpha<1$.

i) If $u \in C_{\bar{u}}^{\alpha}(\Omega)$, then $u \in C_{\partial_{y}}^{\frac{\alpha}{3}}(\Omega)$;

ii) If $u \in C_{\bar{u}}^{1, \alpha}(\Omega)$, then $u \in C_{\partial_{y}}^{\frac{1+\alpha}{3}}(\Omega)$;

iii) If $u \in C_{\bar{u}}^{2, \alpha}(\Omega)$, then $u \in C_{\partial_{y}}^{\frac{2+\alpha}{3}}(\Omega)$;

iv) If $u \in C_{\bar{u}}^{k, \alpha}(\Omega)$, with $k \geq 3$, then there exists $\partial_{y} u$ and it belongs to $C_{\bar{u}}^{k-3, \alpha}(\Omega)$.

Remark 2.13. We explicitly note that $C_{\bar{u}}^{3 k, \alpha}(\Omega) \subseteq C^{k, \frac{\alpha}{3}}(\Omega) \subseteq C_{\bar{u}}^{k, \frac{\alpha}{3}}(\Omega)$, for every $0<\alpha<1$ and $k \geq 1$, where $C^{k, \beta}(\Omega)$ is the space of functions with derivatives up to order $k$ that are $\beta$-Hölder-continuous functions in the Euclidean sense. Thus, if $u \in C_{\bar{u}}^{k, \alpha}(\Omega)$ for every $k \in \mathbb{N}$, then $u \in C^{\infty}(\Omega)$.

Proof of Proposition 2.12. We start with a preliminary remark. If $\bar{u} \in$ $C^{\infty}(\Omega)$, the proof is a consequence of the Campbell-Hausdorff formula. The assertion could be deduced from the general theory also if $\bar{u} \in C_{\bar{u}}^{4, \alpha}(\Omega)$. Since $\bar{u}$ is only of class $C^{1}$, we proceed by direct computation. Let $z_{0}$ be a point in $\Omega$ and, for a suitably small $\delta>0$, let $z_{1}=\exp (\delta X)\left(z_{0}\right), z_{2}=\exp \left(\delta^{2} Y_{\bar{u}}\right)\left(z_{1}\right)$, $z_{3}=\exp (-\delta X)\left(z_{2}\right), z_{4}=\exp \left(-\delta^{2} Y_{\bar{u}}\right)\left(z_{3}\right)$. We claim that

$$
z_{4}=\exp \left(\left(\delta^{3}+o\left(\delta^{3}\right)\right) \bar{u}_{x} \partial_{y}\right)\left(z_{0}\right), \quad \text { as } \delta \rightarrow 0 .
$$

We denote by $\left(x_{j}, y_{j}, t_{j}\right)=z_{j}$, for $j=0,1, \ldots, 4$. Let $\gamma_{j}:[0,1] \rightarrow \Omega$ be the integral path connecting the point $z_{j-1}$ to $z_{j}, j=1,2,3,4$. It is easy to see that

$$
\begin{aligned}
& \gamma_{1}(s)=\left(x_{0}+\delta s, y_{0}, t_{0}\right), \quad \text { then } z_{1}=\left(x_{0}+\delta, y_{0}, t_{0}\right), \\
& \gamma_{2}(s)=\left(x_{0}+\delta, y_{0}+\delta^{2} \int_{0}^{s} \bar{u}\left(\gamma_{2}(\tau)\right) d \tau, t_{0}-\delta^{2} s\right), \\
& \gamma_{3}(s)=\left(x_{0}+\delta(1-s), y_{0}+\delta^{2} \int_{0}^{1} \bar{u}\left(\gamma_{2}(\tau)\right) d \tau, t_{0}-\delta^{2}\right),
\end{aligned}
$$




$$
\gamma_{4}(s)=\left(x_{0}, y_{0}+\delta^{2}\left(\int_{0}^{1} \bar{u}\left(\gamma_{2}(\tau)\right) d \tau-\int_{0}^{s} \bar{u}\left(\gamma_{4}(\tau)\right) d \tau\right), t_{0}+\delta^{2}(s-1)\right)
$$

then it is clear that $x_{4}=x_{0}$ and $t_{4}=t_{0}$. In order to estimate $y_{4}-y_{0}$, we observe that

$$
\begin{aligned}
\bar{u}\left(\gamma_{2}(\tau)\right) & -\bar{u}\left(\gamma_{4}(\tau)\right)=\nabla \bar{u}(\widetilde{z}(\tau)) \cdot\left(\gamma_{2}(\tau)-\gamma_{4}(\tau)\right) \\
& =\delta \bar{u}_{x}(\widetilde{z}(\tau))+\delta^{2} \bar{u}_{y}(\widetilde{z}(\tau))\left(-\int_{\tau}^{1} \bar{u}\left(\gamma_{2}(\sigma)\right) d \sigma+\int_{0}^{\tau} \bar{u}\left(\gamma_{4}(\sigma)\right) d \sigma\right) \\
& +(1-2 \tau) \delta^{2} \bar{u}_{t}(\widetilde{z}(\tau))=\delta \bar{u}_{x}\left(z_{0}\right)(1+o(1)), \quad \text { as } \delta \rightarrow 0
\end{aligned}
$$

since $\bar{u}_{x}$ is a continuous function. As a consequence of (2.15), we get

$$
y_{4}-y_{0}=\delta^{2} \int_{0}^{1}\left(\bar{u}\left(\gamma_{2}(\tau)\right)-\bar{u}\left(\gamma_{4}(\tau)\right)\right) d \tau=\delta^{3} \bar{u}_{x}\left(z_{0}\right)(1+o(1))
$$

as $\delta \rightarrow 0$, then (2.14) is proved. Moreover, since $y_{4}$ depends continuously on $\delta$ and $u_{x}\left(z_{0}\right) \neq 0$, the function $\delta \mapsto y_{4}$ is surjective in a neighborhood of $y_{0}$. Hence, for every $\beta$ sufficiently small, there exists a $\delta=\delta(\beta)$ such that the point $\left(x_{0}, y_{0}+\beta, t_{0}\right)$ can be written as $z_{4}$ in $(2.15)$ (with $\left.\delta=\delta(\beta)\right)$. We stress that (2.17) also yields

$$
\frac{\delta(\beta)^{3}}{\beta} \longrightarrow \frac{1}{\bar{u}_{x}\left(z_{0}\right)} \quad \text { as } \beta \rightarrow 0 .
$$

After these preliminary considerations, we conclude the proof as follows.

(i) Let $\beta$ be chosen as above. Since $u \in C_{\bar{u}}^{\alpha}(\Omega)$, we have

$$
\begin{aligned}
& \left|u\left(z_{0}\right)-u\left(z_{1}\right)\right| \leq C \delta^{\alpha}, \quad\left|u\left(z_{1}\right)-u\left(z_{2}\right)\right| \leq C \delta^{\alpha}, \\
& \left|u\left(z_{2}\right)-u\left(z_{3}\right)\right| \leq C \delta^{\alpha}, \quad\left|u\left(z_{3}\right)-u\left(z_{4}\right)\right| \leq C \delta^{\alpha} ;
\end{aligned}
$$

then, since $\bar{u}_{x}\left(z_{0}\right) \neq 0$,

$$
\left|u\left(z_{0}\right)-u\left(z_{4}\right)\right| \leq 4 C \delta^{\alpha}=4 C\left(\frac{\beta}{\bar{u}_{x}\left(z_{0}\right)(1+o(1))}\right)^{\frac{\alpha}{3}} \quad \text { as } \beta \rightarrow 0,
$$

and this proves (i).

(ii) We consider the functions $\gamma_{j}:[0,1] \rightarrow \mathbb{R}$, defined in (2.15) for $j=$ $1, \ldots, 4$ and we apply the Taylor expansion of first order to $u \circ \gamma_{j}$. Since, by hypothesis, $u$ is of class $C^{1, a}$ as a function of the first variable $x$, we have

$$
\begin{aligned}
& u\left(z_{0}\right)-u\left(z_{1}\right)=\delta u_{x}\left(z_{1}\right)+O\left(\delta^{\alpha+1}\right), \\
& u\left(z_{2}\right)-u\left(z_{3}\right)=-\delta u_{x}\left(z_{2}\right)+O\left(\delta^{\alpha+1}\right) .
\end{aligned}
$$


Since $u \in C_{Y_{\bar{u}}}^{\frac{1+\alpha}{2}}(\Omega)$,

$$
\left|u\left(z_{1}\right)-u\left(z_{2}\right)\right| \leq C \delta^{\alpha+1}, \quad\left|u\left(z_{3}\right)-u\left(z_{4}\right)\right| \leq C \delta^{\alpha+1} .
$$

Then, by using $(2.20),(2.18)$ and the fact that $u_{x} \in C_{\bar{u}}^{\alpha}(\Omega)$, we conclude that $u\left(z_{0}\right)-u\left(z_{4}\right)=O\left(\delta^{\alpha+1}\right)=O\left(\beta^{\frac{\alpha+1}{3}}\right)$ as $\beta \rightarrow 0$.

(iii) In this case we use Taylor polynomials of order 2 . As $\delta$ tends to zero, we have

$$
\begin{aligned}
& u\left(z_{0}\right)-u\left(z_{1}\right)=\delta u_{x}\left(z_{1}\right)+\frac{\delta^{2}}{2} u_{x x}\left(z_{1}\right)+O\left(\delta^{2+\alpha}\right), \\
& u\left(z_{2}\right)-u\left(z_{3}\right)=-\delta u_{x}\left(z_{2}\right)-\frac{\delta^{2}}{2} u_{x x}\left(z_{2}\right)+O\left(\delta^{2+\alpha}\right), \\
& u\left(z_{1}\right)-u\left(z_{2}\right)=\delta^{2} Y_{\bar{u}} u\left(z_{2}\right)+O\left(\delta^{\alpha+2}\right), \\
& u\left(z_{3}\right)-u\left(z_{4}\right)=-\delta^{2} Y_{\bar{u}} u\left(z_{3}\right)+O\left(\delta^{\alpha+2}\right) .
\end{aligned}
$$

Hence we obtain

$$
\begin{aligned}
u\left(z_{0}\right)-u\left(z_{4}\right) & =\delta\left(u_{x}\left(z_{1}\right)-u_{x}\left(z_{2}\right)\right)+\frac{\delta^{2}}{2}\left(u_{x x}\left(z_{1}\right)-u_{x x}\left(z_{2}\right)\right) \\
& +\delta^{2}\left(Y_{\bar{u}} u\left(z_{2}\right)-Y_{\bar{u}} u\left(z_{3}\right)\right)+O\left(\delta^{2+\alpha}\right)
\end{aligned}
$$

(since $u_{x} \in C_{\bar{u}}^{1, \alpha}(\Omega)$ and $\left.u_{x x}, Y_{\bar{u}} \in C_{\bar{u}}^{\alpha}(\Omega)\right)=O\left(\delta^{2+\alpha}\right)$, as $\delta \rightarrow 0$, and this yields (iii).

(iv) We first consider the problem for $k=3$. By using a Taylor polynomial of higher order, we get, as $\delta$ tends to zero,

$$
\begin{aligned}
& u\left(z_{0}\right)-u\left(z_{1}\right)=\delta u_{x}\left(z_{1}\right)+\frac{\delta^{2}}{2} u_{x x}\left(z_{1}\right)+\frac{\delta^{3}}{6} u_{x x x}\left(z_{1}\right)+O\left(\delta^{3+\alpha}\right), \\
& u\left(z_{1}\right)-u\left(z_{2}\right)=-\delta^{2} Y_{\bar{u}} u\left(z_{2}\right)+O\left(\delta^{3+\alpha}\right), \\
& u\left(z_{2}\right)-u\left(z_{3}\right)=-\delta u_{x}\left(z_{2}\right)-\frac{\delta^{2}}{2} u_{x x}\left(z_{2}\right)-\frac{\delta^{3}}{6} u_{x x x}\left(z_{2}\right)+O\left(\delta^{3+\alpha}\right), \\
& u\left(z_{3}\right)-u\left(z_{4}\right)=\delta^{2} Y_{\bar{u}} u\left(z_{3}\right)+O\left(\delta^{3+\alpha}\right) .
\end{aligned}
$$

Then

$$
\begin{aligned}
u\left(z_{0}\right) & -u\left(z_{4}\right)=\delta\left(u_{x}\left(z_{1}\right)-u_{x}\left(z_{2}\right)\right)+\frac{\delta^{2}}{2}\left(u_{x x}\left(z_{1}\right)-u_{x x}\left(z_{2}\right)\right) \\
& +\delta^{2}\left(Y_{\bar{u}} u\left(z_{2}\right)-Y_{\bar{u}} u\left(z_{3}\right)\right)+\frac{\delta^{3}}{6}\left(u_{x x x}\left(z_{1}\right)-u_{x x x}\left(z_{2}\right)\right)+O\left(\delta^{3+\alpha}\right) .
\end{aligned}
$$

Since $u_{x} \in C_{\bar{u}}^{2, \alpha}(\Omega), Y_{\bar{u}} u_{x} \in C_{\bar{u}}^{\alpha}(\Omega)$ and $d_{\bar{u}}\left(z_{0}, z_{2}\right) \leq \delta$, we have

$$
u_{x}\left(z_{1}\right)-u_{x}\left(z_{2}\right)=-\delta^{2} Y_{\bar{u}} u_{x}\left(z_{2}\right)+O\left(\delta^{2+\alpha}\right)=-\delta^{2} Y_{\bar{u}} u_{x}\left(z_{0}\right)+O\left(\delta^{2+\alpha}\right) .
$$


Since $u_{x x} \in C_{\bar{u}}^{\frac{1+\alpha}{2}}(\Omega)$ and $u_{x x x} \in C_{\bar{u}}^{\alpha}(\Omega)$, we have

$$
u_{x x}\left(z_{1}\right)-u_{x x}\left(z_{2}\right)=O\left(\delta^{1+\alpha}\right), \quad u_{x x x}\left(z_{1}\right)-u_{x x x}\left(z_{2}\right)=O\left(\delta^{\alpha}\right) .
$$

Moreover, by the fact that $Y_{\bar{u}} u \in C_{\bar{u}}^{1, \alpha}(\Omega), \partial_{x} Y_{\bar{u}} u \in C_{\bar{u}}^{\alpha}(\Omega)$ and $d_{\bar{u}}\left(z_{0}, z_{2}\right) \leq$ $\delta$, we get

$$
Y_{\bar{u}} u\left(z_{2}\right)-Y_{\bar{u}} u\left(z_{3}\right)=\delta \partial_{x} Y_{\bar{u}} u\left(z_{2}\right)+O\left(\delta^{1+\alpha}\right)=\delta \partial_{x} Y_{\bar{u}} u\left(z_{0}\right)+O\left(\delta^{1+\alpha}\right) .
$$

Inserting in (2.22), we finally obtain

$$
u\left(z_{0}\right)-u\left(z_{4}\right)=\delta^{3}\left(\partial_{x} Y_{\bar{u}} u\left(z_{0}\right)-Y_{\bar{u}} u_{x}\left(z_{0}\right)\right)+O\left(\delta^{3+\alpha}\right)
$$

or, in other words,

$$
\begin{gathered}
\frac{u\left(x_{0}, y_{0}+\beta, t_{0}\right)-u\left(x_{0}, y_{0}, t_{0}\right)}{\beta}=\frac{\delta(\beta)^{3}}{\beta}\left[X, Y_{\bar{u}}\right] u\left(z_{0}\right)+O\left(\delta(\beta)^{\alpha}\right) \\
\longrightarrow \frac{1}{\bar{u}_{x}\left(z_{0}\right)}\left[X, Y_{\bar{u}}\right] u\left(z_{0}\right), \quad \text { as } \beta \rightarrow 0 .
\end{gathered}
$$

This proves the existence of $\partial_{y} u$. The regularity follows from the fact that

$$
u_{y}(z)=\frac{1}{\bar{u}_{x}\left(z_{0}\right)}\left(\partial_{x} Y_{\bar{u}} u(z)-Y_{\bar{u}} u_{x}(z)\right), \quad \forall z \in \Omega .
$$

The proof in the case $k>3$ is immediate: the existence of $\partial_{y} u$ has been proved, while its regularity directly follows from the above identity.

We introduce the "Taylor polynomials" related to the spaces $C_{\bar{u}}^{k, \alpha}(\Omega)$ above considered.

Definition 2.14. Let $z_{0}=\left(x_{0}, y_{0}, t_{0}\right) \in \Omega, k \in \mathbb{N}$ and let $\bar{u}$ be a $C^{1}$ function such that (1.2) holds. We denote by $P_{z_{0}}^{k}$ any function of the form

$$
P_{z_{0}}^{k}(x, y, t)=\sum_{i+2 j+3 m \leq k} c_{i, j, m}\left(x-x_{0}\right)^{i}\left(t-t_{0}\right)^{j}\left(y-y_{0}+\left(t-t_{0}\right) \bar{u}\left(z_{0}\right)\right)^{m}
$$

where $i, j, m \in \mathbb{N} \cup\{0\}$ and $c_{i, j, m}$ are real constants. We say that $P_{z_{0}}^{k}$ is a polynomial of initial point $z_{0}$ and $\delta_{\lambda}^{\left(z_{0}\right)}$-degree $k$.

Remark 2.15. The functions $x-x_{0}, t-t_{0}$ and $y-y_{0}+\left(t-t_{0}\right) \bar{u}\left(z_{0}\right)$ are $\delta_{\lambda}^{\left(z_{0}\right)}$-homogeneous of degree 1,2 and 3 , respectively, since they are $\theta_{1},-\theta_{2}$ and $\bar{u}_{x}\left(z_{0}\right)\left(\theta_{3}+\theta_{1} \theta_{2}\right)$, where $\left(\theta_{1}, \theta_{2}, \theta_{3}\right) \equiv \theta_{z_{0}}^{\left(z_{0}\right)}(z)$ defined in $(2.8)$.

We next state the main result of this subsection. 
Theorem 2.16. Let $z_{0} \in \Omega, 0<\alpha<1, k \in \mathbb{N} \cup\{0\}$ and assume that $u \in C_{\bar{u}}^{k, \alpha}(\Omega)$. Then there exists a polynomial function $P_{z_{0}}^{k} u$, of $\delta_{\lambda}^{\left(z_{0}\right)}$-degree $k$, such that

$$
u(z)=P_{z_{0}}^{k} u(z)+O\left(d_{\bar{u}}\left(z_{0}, z\right)^{k+\alpha}\right) \quad \text { as } z \rightarrow z_{0} .
$$

Proof. We prove our result by a classical argument that relies on Proposition 2.12. We observe that the regularity assumption is given in terms of the geometry corresponding to $\theta_{\bar{u}, z_{0}}$ while we obtain polynomial functions that are homogeneous with respect to $\delta_{\lambda}^{\left(z_{0}\right)}$.

The assertion is obvious if $k=0$. We first prove (2.25) for $k=1,2$. We proceed essentially as in the proof of Proposition 2.12. We consider a point $z_{0}=\left(x_{0}, y_{0}, t_{0}\right) \in \Omega$ and a compact neighborhood $K \subset \Omega$ of $z_{0}$ such that every $z=(x, y, t) \in K$ can be connected to $z_{0}$ as follows. Let

$$
\begin{aligned}
& \gamma_{1}(s)=\exp \left(s\left(x-x_{0}\right) X\right)\left(z_{0}\right) \quad \text { and } \quad z_{1}=\gamma_{1}(1)=\left(x, y_{0}, t_{0}\right) \\
& \gamma_{2}(s)=\exp \left(-s\left(t-t_{0}\right) Y_{\bar{u}}\right)\left(z_{1}\right) \quad \text { and } \quad z_{2}=\gamma_{2}(1)=\left(x, y_{2}, t\right) \\
& \gamma_{3}(s)=\exp \left(s\left(y-y_{2}\right) \partial_{y}\right)\left(z_{2}\right) .
\end{aligned}
$$

Clearly

$$
\left|x-x_{0}\right| \leq d_{\bar{u}}\left(z_{0}, z\right), \quad\left|t-t_{0}\right| \leq d_{\bar{u}}\left(z_{0}, z\right)^{2} .
$$

Let us now estimate $\left|y-y_{2}\right|$. We have

$$
\begin{aligned}
\left|y-y_{2}\right| & =d_{\bar{u}}\left(z, z_{2}\right)^{3} \leq \widetilde{C}^{2}\left(d_{\bar{u}}\left(z, z_{0}\right)+d_{\bar{u}}\left(z_{0}, z_{1}\right)+d_{\bar{u}}\left(z_{1}, z_{2}\right)\right)^{3} \\
& \leq \widetilde{C}^{2}\left(d_{\bar{u}}\left(z, z_{0}\right)+\left|x-x_{0}\right|+\left|t-t_{0}\right|^{\frac{1}{2}}\right)^{3} \leq C d_{\bar{u}}\left(z_{0}, z\right)^{3} .
\end{aligned}
$$

Let us give a detailed proof of $(2.25)$ for $k=1$. The case $k=2$ can be treated analogously.

$$
u(z)-u\left(z_{0}\right)=u\left(z_{1}\right)-u\left(z_{0}\right)+u\left(z_{2}\right)-u\left(z_{1}\right)+u(z)-u\left(z_{2}\right)=
$$

(since $u \in C^{1, \alpha}(\Omega)$ as a function of its first variable, $u \in C_{Y_{\bar{u}}}^{\frac{1+\alpha}{2}}(\Omega)$ and $\left.u \in C_{\partial_{y}}^{\frac{2+\alpha}{3}}(\Omega)\right)$

$=u\left(z_{0}\right)+\left(x-x_{0}\right) u_{x}\left(z_{0}\right)+O\left(d_{\bar{u}}\left(z_{0}, z\right)^{1+\alpha}\right)+O\left(\left|t-t_{0}\right|^{\frac{1+\alpha}{2}}\right)+O\left(\left|y-y_{2}\right|^{\frac{2+\alpha}{3}}\right)=$

(by (2.26) and (2.27))

$$
=u\left(z_{0}\right)+\left(x-x_{0}\right) u_{x}\left(z_{0}\right)+O\left(d_{\bar{u}}\left(z_{0}, z\right)^{1+\alpha}\right), \quad \text { as } z \rightarrow z_{0} .
$$

For $k \geq 3$ we simply argue by induction. We recall that, by our convention, we assume $\bar{u} \in C_{\bar{u}}^{k-2, \alpha}(\Omega)$. If $\widetilde{\gamma}$ is the Euclidean segment connecting $z$ and 
$z_{0}$, we have

$$
\begin{aligned}
u(z)-u\left(z_{0}\right) & =\left(x-x_{0}\right) \int_{0}^{1} \partial_{x} u(\widetilde{\gamma}(s)) d s-\left(t-t_{0}\right) \int_{0}^{1} Y_{\bar{u}} u(\widetilde{\gamma}(s)) d s \\
& +\left(y-y_{0}+\bar{u}\left(z_{0}\right)\left(t-t_{0}\right)\right) \int_{0}^{1} \partial_{y} u(\widetilde{\gamma}(s)) d s \\
& +\left(t-t_{0}\right) \int_{0}^{1}\left(\bar{u}(\widetilde{\gamma}(s))-\bar{u}\left(z_{0}\right)\right) \partial_{y} u(\widetilde{\gamma}(s)) d s .
\end{aligned}
$$

By the inductive hypothesis, $u_{x}$ has a Taylor expansion of the form (2.24)(2.25) of $\delta_{\lambda}^{\left(z_{0}\right)}$-degree $k-1$. Thus we have

$$
\begin{aligned}
& \left(x-x_{0}\right) \int_{0}^{1} \partial_{x} u(\widetilde{\gamma}(s)) d s=\left(x-x_{0}\right) \int_{0}^{1} \sum_{i+2 j+3 m \leq k-1} c_{i, j, m}\left(x-x_{0}\right)^{i}\left(t-t_{0}\right)^{j} \\
& \times\left(y-y_{0}+\left(t-t_{0}\right) \bar{u}\left(z_{0}\right)\right)^{m} s^{i+j+m} d s+\left(x-x_{0}\right) \int_{0}^{1} O\left(d_{\bar{u}}\left(z_{0}, \widetilde{\gamma}(s)\right)^{k-1+\alpha}\right) d s \\
& =\sum_{i+2 j+3 m \leq k-1} \frac{c_{i, j, m}}{i+j+m+1}\left(x-x_{0}\right)^{i+1}\left(t-t_{0}\right)^{j}\left(y-y_{0}+\left(t-t_{0}\right) \bar{u}\left(z_{0}\right)\right)^{m} \\
& +O\left(d_{\bar{u}}\left(z_{0}, z\right)^{k+\alpha}\right), \quad \text { as } z \rightarrow z_{0} .
\end{aligned}
$$

In the same way we can handle the second and the third term in the righthand side of (2.28), since, by our assumption, $Y_{\bar{u}} u \in C_{\bar{u}}^{k-2, \alpha}(\Omega)$ and $\partial_{y} u \in$ $C_{\bar{u}}^{k-3, \alpha}(\Omega)$. The last term can be estimated as follows. Let $\bar{c}_{i, j, m}$ (respectively $\hat{c}_{i, j, m}$ ) denote the coefficients of $P_{z_{0}}^{k-2} \bar{u}$ (respectively $P_{z_{0}}^{k-3} u_{y}$ ). Then we have

$$
\begin{aligned}
& \quad\left(t-t_{0}\right) \int_{0}^{1}\left(\bar{u}(\widetilde{\gamma}(s))-\bar{u}\left(z_{0}\right)\right) \partial_{y} u(\widetilde{\gamma}(s)) d s \\
& =\left(t-t_{0}\right) \int_{0}^{1}\left(P_{z_{0}}^{k-2} \bar{u}(\widetilde{\gamma}(s))-\bar{u}\left(z_{0}\right)+O\left(d_{\bar{u}}\left(z_{0}, \widetilde{\gamma}(s)\right)^{k-2+\alpha}\right)\right) \\
& \quad \cdot\left(P_{z_{0}}^{k-3} u_{y}(\widetilde{\gamma}(s))+O\left(d_{\bar{u}}\left(z_{0}, \widetilde{\gamma}(s)\right)^{k-3+\alpha}\right)\right) d s \\
& \left(\operatorname{since} P_{z_{0}}^{k-2} \bar{u}(\widetilde{\gamma}(s))-\bar{u}\left(z_{0}\right)=O\left(d_{\bar{u}}\left(z_{0}, z\right)\right), \text { as } z \rightarrow z_{0}\right) \\
& =\int_{0}^{1} \sum_{\substack{0<i+2 j+3 m \leq k-2, i_{1}+j_{1}+m_{1} \leq k-3}} \bar{c}_{i, j, m} \hat{c}_{i_{1}, j_{1}, m_{1}}\left(x-x_{0}\right)^{i+i_{1}}\left(t-t_{0}\right)^{j+j_{1}+1} \cdot \\
& \quad \cdot\left(y-y_{0}+\left(t-t_{0}\right) \bar{u}\left(z_{0}\right)\right)^{m+m_{1}} s^{i+i_{1}+j+j_{1}+m+m_{1}} d s+O\left(d_{\bar{u}}\left(z_{0}, z\right)^{k+\alpha}\right) .
\end{aligned}
$$


2.3. Parametrix. In this subsection, we provide some results about the fundamental solution of the frozen operator $L_{z_{0}}$. As we previously noticed, the second-order differential operator

$$
L_{H}=X_{H}^{2}+Y_{H}
$$

has a fundamental solution $\Gamma_{H}$, which is invariant with respect to the left $\oplus$-translations and $\delta_{\lambda}^{H}$-homogeneous of degree $-Q+2$. Hence a fundamental solution of $L_{z_{0}}$ is given by

$$
\Gamma_{z_{0}}(z, \zeta)=\frac{1}{\bar{u}_{x}\left(z_{0}\right)} \Gamma_{H}\left(\left(-\theta_{z_{0}}^{\left(z_{0}\right)}(\zeta)\right) \oplus \theta_{z_{0}}^{\left(z_{0}\right)}(z)\right)
$$

and it is $\delta_{\lambda}^{\left(z_{0}\right)}$-homogeneous of degree $-Q+2$. We remark that in [22], Kolmogorov wrote explicitly the fundamental solution of the operator

$$
\partial_{x x}+x \partial_{y}-\partial_{t}
$$

which is, up to a canonical change of coordinates, the fundamental solution of $L_{H}$ or $L_{z_{0}}$. However here we don't make use of that explicit formula, but we use only its local behavior.

For the sake of convenience, here and in the sequel we systematically use the following notation:

$$
\begin{aligned}
& D_{1}=X, \quad D_{1}^{\left(z_{0}\right)}=X, \quad D_{1}^{H}=X_{H}, \\
& D_{2}=Y_{\bar{u}}, \quad D_{2}^{\left(z_{0}\right)}=Y_{z_{0}}, \quad D_{2}^{H}=Y_{H} \text {. }
\end{aligned}
$$

Besides we denote $D_{3}=\partial_{y}, D_{3}^{\left(z_{0}\right)}=\bar{u}_{x}\left(z_{0}\right) \partial_{y}, D_{3}^{H}=\partial_{\theta_{3}}$. We also denote the identity by $D_{0}=D_{0}^{\left(z_{0}\right)}=D_{0}^{H}$. For every multi-index $\sigma=\left(\sigma_{1}, \ldots, \sigma_{m}\right)$, with $\sigma_{r} \in\{0,1,2,3\}, 1 \leq r \leq m \in \mathbb{N}$, we set

$$
D_{\sigma}=D_{\sigma_{1}} \cdots D_{\sigma_{m}}, \quad D_{\sigma}^{\left(z_{0}\right)}=D_{\sigma_{1}}^{\left(z_{0}\right)} \cdots D_{\sigma_{m}}^{\left(z_{0}\right)}, \quad D_{\sigma}^{H}=D_{\sigma_{1}}^{H} \cdots D_{\sigma_{m}}^{H} .
$$

We call height of $\sigma$ the natural number

$$
|\sigma|=\sum_{r=1}^{m} \sigma_{r} .
$$

We remark that $D_{\sigma}^{\left(z_{0}\right)}$ (resp. $D_{\sigma}^{H}$ ) is a $\delta_{\lambda}^{\left(z_{0}\right)}$ (respectively $\delta_{\lambda}^{H}$ )-homogeneous operator of degree $|\sigma|$. Since $\Gamma_{z_{0}}$ depends on many variables, the notation $D\left(z_{1}\right) \Gamma_{z_{0}}(\cdot, \zeta)$ shall denote the $D$-derivative of $\Gamma_{z_{0}}(z, \zeta)$ with respect to the variable $z$, evaluated at the point $z_{1}$.

If $\varphi \in C_{0}^{\infty}$, a simple relation holds between the derivatives $D_{\sigma} \varphi$ and $D_{\sigma}^{\left(z_{0}\right)} \varphi$. 
Lemma 2.17. If $\bar{u} \in C_{\bar{u}}^{k, \alpha}(\Omega)$, and $\sigma \in\{0,1,2\}^{k+1}$ with $|\sigma| \leq k+1$, then for every function $\varphi \in C_{0}^{\infty}(\Omega)$ the derivative $D_{\sigma} \varphi$ can be represented as

$$
D_{\sigma} \varphi(z)=\sum_{\varrho \in I_{\sigma}} C_{\varrho} \frac{\left(\bar{u}-P_{z_{0}}^{1} \bar{u}\right)^{k_{\varrho}}(z)}{\left(\bar{u}_{x}\left(z_{0}\right)\right)^{h_{\varrho}}} \prod_{\mu \in J_{\varrho}} D_{\mu} \bar{u}(z) D_{\varrho}^{\left(z_{0}\right)} \varphi(z),
$$

where $I_{\sigma}$ and $J_{\varrho}$ are suitable subsets of $\{0,1,2,3\}^{k+1}, C_{\varrho}$ are nonnegative constants, and $h_{\varrho}$ and $k_{\varrho}$ are nonnegative integers such that

$$
|\varrho| \leq|\sigma|+k_{\varrho}, \quad k_{\varrho} \leq h_{\varrho}, \quad|\mu| \leq k_{\varrho} \leq 3|\sigma|, \quad \forall \mu \in I_{\varrho}, \forall \varrho \in I_{\sigma} .
$$

If $J_{\varrho}$ is empty, we set $\prod_{\mu \in J_{\varrho}} D_{\sigma} \bar{u}=1$.

Proof. Since the function $\varphi$ is of class $C_{0}^{\infty}(\Omega)$, by Remark 2.8 its Lie derivatives can be represented in terms of the standard partial derivatives, and it is not necessary to use the exponential function. If $|\sigma| \leq 2$, the assertion follows directly from the definition. Indeed, if $\sigma \in\{(1),(1,1)\}$, then $D_{\sigma}=D_{\sigma}^{\left(z_{0}\right)}$, while, if $\sigma=(2)$, then

$$
D_{\sigma}=D_{\sigma}^{\left(z_{0}\right)}+\frac{\left(\bar{u}-P_{z_{0}}^{1} \bar{u}\right)}{\bar{u}_{x}\left(z_{0}\right)} D_{3}^{\left(z_{0}\right)} .
$$

The general assertion follows by induction on $|\sigma|$.

Analogously it is not difficult to prove the following.

Lemma 2.18. If $\bar{u} \in C_{\bar{u}}^{1, \alpha}(\Omega), \sigma \in\{0,1,2\}^{k+1}$ with $|\sigma| \leq k+1$, and $z_{0}$, $z_{1} \in \Omega$, then for every function $\varphi \in C_{0}^{\infty}(\Omega)$

$D_{\sigma}^{\left(z_{1}\right)} \varphi(z)=$

$D_{\sigma}^{\left(z_{0}\right)} \varphi(z)+\sum_{\varrho \in J_{\sigma}} C_{\varrho}\left(\bar{u}-P_{z_{1}}^{1} \bar{u}\right)^{k_{\varrho}}\left(z_{0}\right)\left(x-x_{0}\right)^{h_{\varrho}}\left(\bar{u}_{x}\left(z_{0}\right)-\bar{u}_{x}\left(z_{1}\right)\right)^{j_{\varrho}} D_{\varrho}^{\left(z_{0}\right)} \varphi(z)$,

where $J_{\sigma}$ is a suitable family of subsets of $\{0,1,2,3\}^{k+1}, C_{\varrho}$ are nonnegative constants, and $j_{\varrho}$ and $k_{\varrho}$ are nonnegative integers such that

$$
|\varrho| \leq|\sigma|+k_{\varrho}+h_{\varrho} \quad h_{\varrho} \leq j_{\varrho}, \forall \varrho \in I_{\sigma} .
$$

Proof. If $|\sigma| \in\{(1),(1,1)\}$ the assertion is obvious. If $\sigma=(2)$, then

$$
\begin{aligned}
D_{\sigma}^{\left(z_{1}\right)} \varphi(z) & -D_{\sigma}^{\left(z_{0}\right)} \varphi(z)=\left(P_{z_{1}}^{1} \bar{u}(z)-P_{z_{0}}^{1} \bar{u}(z)\right) \partial_{y} \varphi(z) \\
& =-\left(\bar{u}\left(z_{0}\right)-P_{z_{1}}^{1} \bar{u}\left(z_{0}\right)-\left(x-x_{0}\right)\left(\bar{u}_{x}\left(z_{1}\right)-\bar{u}_{x}\left(z_{0}\right)\right)\right) \partial_{y} \varphi(z) .
\end{aligned}
$$


Now, let us suppose that the assertion is true for every multi-index of height less than or equal to $k-1$. We choose $\sigma=\left(\sigma_{1}, \sigma^{\prime}\right)$, of height $|\sigma|=k$. We assume for simplicity that $\sigma_{1}=1$, since the proof is similar if $\sigma_{1}=2$. Then

$$
\begin{aligned}
& D_{\sigma}^{\left(z_{1}\right)} \varphi= \\
& D_{1}^{\left(z_{0}\right)}\left(D_{\sigma^{\prime}}^{\left(z_{0}\right)} \varphi+\sum_{\varrho \in J_{\sigma^{\prime}}} C_{\varrho}\left(\bar{u}-P_{z_{1}}^{1} \bar{u}\right)^{k_{\varrho}}\left(z_{0}\right)\left(x-x_{0}\right)^{h_{\varrho}}\left(\bar{u}_{x}\left(z_{0}\right)-\bar{u}_{x}\left(z_{1}\right)\right)^{j_{\varrho}} D_{\varrho}^{\left(z_{0}\right)} \varphi\right) \\
& =D_{\sigma}^{\left(z_{0}\right)} \varphi+\sum_{\varrho \in J_{\sigma^{\prime}}} h_{\varrho} C_{\varrho}\left(\bar{u}-P_{z_{1}}^{1} \bar{u}\right)^{k_{\varrho}}\left(z_{0}\right)\left(x-x_{0}\right)^{h_{\varrho}-1}\left(\bar{u}_{x}\left(z_{0}\right)-\bar{u}_{x}\left(z_{1}\right)\right)^{j_{\varrho}} D_{\varrho}^{\left(z_{0}\right)} \varphi \\
& +\sum_{\varrho \in J_{\sigma^{\prime}}} C_{\varrho}\left(\bar{u}-P_{z_{1}}^{1} \bar{u}\right)^{k_{\varrho}}\left(z_{0}\right)\left(x-x_{0}\right)^{h_{\varrho}}\left(\bar{u}_{x}\left(z_{0}\right)-\bar{u}_{x}\left(z_{1}\right)\right)^{j_{\varrho}} D_{1}^{\left(z_{0}\right)} D_{\varrho}^{\left(z_{0}\right)} \varphi .
\end{aligned}
$$

Remark 2.19. It is well known that for every compact set $K \subset \Omega$ and for every multi-index $\sigma$, there exists a positive constant $C$ such that

$$
\left|D_{\sigma}^{\left(z_{0}\right)}(z) \Gamma_{z_{0}}(\cdot, \zeta)\right| \leq C d_{z_{0}}(z, \zeta)^{-Q+2-|\sigma|}, \quad \forall z, z_{0}, \zeta \in K, z \neq \zeta .
$$

By Lemma 2.17, we also have

$$
\left|D_{\sigma}(z) \Gamma_{z_{0}}(\cdot, \zeta)\right| \leq C d_{z_{0}}(z, \zeta)^{-Q+2-|\sigma|} .
$$

Fixing an open subset $\Omega$ of $\mathbb{R}^{3}$, a positive constant $M$ and two points $z_{0}, z \in \Omega$, we set

$$
\Omega_{M}=\left\{\zeta \in \Omega: d_{z_{0}}\left(z_{0}, \zeta\right) \geq M d_{z_{0}}\left(z_{0}, z\right)\right\} .
$$

This set is defined in such a way that the function $z \mapsto \Gamma_{z_{0}}(z, \zeta)$ is smooth, if $\zeta$ belongs to $\Omega_{M}$. Indeed, if $M$ is sufficiently large, then, by (2.6),

$$
d_{z_{0}}(z, \zeta) \geq \frac{1}{\widetilde{C}} d_{z_{0}}\left(z_{0}, \zeta\right)-d_{z_{0}}\left(z_{0}, z\right) \geq\left(\frac{1}{\widetilde{C}}-\frac{1}{M}\right) d_{z_{0}}\left(z_{0}, \zeta\right)
$$

for every $\zeta \in \Omega_{M}$.

Proposition 2.20. Let $k \in \mathbb{N}$. Let $\bar{u} \in C_{\bar{u}}^{k-1, \alpha}(\Omega)$ and let $K$ be a compact subset of $\Omega$. There is a positive constant $C$, such that, for every multi-index $\sigma,|\sigma|=k$, we have

$$
\begin{aligned}
& \left|\left(D_{\sigma}(z)-D_{\sigma}\left(z_{0}\right)\right) \Gamma_{z_{0}}(\cdot, \zeta)\right| \\
& \leq C\left(d_{z_{0}}\left(z_{0}, z\right) d_{z_{0}}\left(z_{0}, \zeta\right)^{-Q+1-k}+d_{z_{0}}\left(z_{0}, z\right)^{\alpha} d_{z_{0}}\left(z_{0}, \zeta\right)^{-Q+2-k}\right),
\end{aligned}
$$

for every $z, z_{0} \in K, \zeta \in \Omega_{M}$ defined in (2.33) for suitable $M>0$. 
Proof. We apply Lemma 2.17, and denote $\widetilde{I}_{\sigma}=\left\{\varrho: k_{\varrho}=0\right\}$. Then, we have

$$
\begin{aligned}
& \left(D_{\sigma}(z)-D_{\sigma}\left(z_{0}\right)\right) \Gamma_{z_{0}}(\cdot, \zeta) \\
& =\sum_{\varrho \in I_{\sigma} \backslash \widetilde{I}_{\sigma}} C_{\varrho} \frac{\left(\bar{u}-P_{z_{0}}^{1} \bar{u}\right)^{k_{\varrho}}(z)}{\left(\bar{u}_{x}\left(z_{0}\right)\right)^{h_{\varrho}}} \prod_{\mu \in J_{\varrho}} D_{\mu} \bar{u}(z) D_{\varrho}^{\left(z_{0}\right)}(z) \Gamma_{z_{0}}(\cdot, \zeta) \\
& +\sum_{\varrho \in \widetilde{I}_{\sigma}} C_{\varrho}\left(\prod_{\mu \in J_{\varrho}} D_{\mu} \bar{u}(z)-\prod_{\mu \in J_{\varrho}} D_{\mu} \bar{u}\left(z_{0}\right)\right) D_{\varrho}^{\left(z_{0}\right)}\left(z_{0}\right) \Gamma_{z_{0}}(\cdot, \zeta) \\
& +\sum_{\varrho \in \widetilde{I}_{\sigma}} C_{\varrho} \prod_{\mu \in J_{\varrho}} D_{\mu} \bar{u}(z)\left(D_{\varrho}^{\left(z_{0}\right)}(z)-D_{\varrho}^{\left(z_{0}\right)}\left(z_{0}\right)\right) \Gamma_{z_{0}}(\cdot, \zeta) .
\end{aligned}
$$

Now we estimate each term separately. By simplicity let us call them $S_{1}$, $S_{2}, S_{3}$ respectively. We first note that if with $M>\widetilde{C}, \zeta \in \Omega_{M}$ and $\bar{z} \in \Omega$ is such that $d_{z_{0}}\left(z_{0}, \bar{z}\right) \leq d_{z_{0}}\left(z_{0}, z\right)$, then we have

$$
d_{z_{0}}(\bar{z}, \zeta) \geq \frac{1}{\widetilde{C}} d_{z_{0}}\left(z_{0}, \zeta\right)-d_{z_{0}}\left(z_{0}, \bar{z}\right) \geq \frac{1}{\widetilde{C}} d_{z_{0}}\left(z_{0}, \zeta\right)-d_{z_{0}}\left(z_{0}, z\right)
$$

(by definition of $\Omega_{M}$ )

$$
\geq\left(\frac{1}{\widetilde{C}}-\frac{1}{M}\right) d_{z_{0}}\left(z_{0}, \zeta\right)
$$

We first consider $S_{1}$

$$
\left|S_{1}\right| \leq C \sum_{|\varrho| \leq|\sigma|+k_{\varrho}} d_{z_{0}}\left(z_{0}, z\right)^{k_{\varrho}(1+\alpha)} d_{z_{0}}(z, \zeta)^{-Q+2-|\varrho|} \leq
$$

(using (2.33), (2.34), the fact that $|\varrho| \leq|\sigma|+k_{\varrho}$ and that $K$ is bounded)

$$
\leq C d_{z_{0}}\left(z_{0}, z\right)^{\alpha} d_{z_{0}}\left(z_{0}, \zeta\right)^{-Q+2-|\sigma|} .
$$

Analogously we can estimate $S_{2}$. Indeed, since $D_{\mu} \bar{u} \in C^{\alpha}$ for every $\mu$ such that $|\mu| \leq k$ and $|\varrho| \leq|\sigma|$, we get

$$
\left|S_{2}\right| \leq C d_{z_{0}}\left(z_{0}, z\right)^{\alpha} d_{z_{0}}\left(z_{0}, \zeta\right)^{-Q+2-|\sigma|} .
$$

Finally

$$
\left|S_{3}\right| \leq \sum_{|\varrho| \leq|\sigma|} C_{\varrho} \prod_{\mu \in I_{\varrho}} D_{\mu} \bar{u}(z)\left(D_{\varrho}^{\left(z_{0}\right)}(z)-D_{\varrho}^{\left(z_{0}\right)}\left(z_{0}\right)\right) \Gamma_{z_{0}}(\cdot, \zeta)=
$$

(by the mean value theorem, for some $\bar{z}$ such that $d_{z_{0}}\left(z_{0}, \bar{z}\right) \leq d_{z_{0}}\left(z_{0}, z\right)$ )

$$
=\sum_{|\varrho| \leq|\sigma|} C_{\varrho}\left\langle\theta_{z_{0}}^{\left(z_{0}\right)}(z),\left(\nabla_{z_{0}} D_{\varrho}^{\left(z_{0}\right)}\right)(\bar{z}) \Gamma_{z_{0}}(\cdot, \zeta)\right\rangle
$$




$$
\leq \sum_{|\varrho| \leq|\sigma|} C_{\varrho} \sum_{r=1}^{3} d_{z_{0}}\left(z_{0}, z\right)^{r} d_{z_{0}}(\bar{z}, \zeta)^{-Q-r+2-|\varrho|}
$$

(by (2.36), and the definition of $\Omega_{M}$ )

$$
\leq C d_{z_{0}}\left(z_{0}, z\right) d_{z_{0}}\left(z_{0}, \zeta\right)^{-Q+1-|\sigma|} .
$$

The main results of this section are contained in the following statement.

Proposition 2.21. Let $k \in \mathbb{N}, \bar{u} \in C_{\bar{u}}^{k-1, \alpha}(\Omega)$ and $K$ be a compact subset of $\Omega$. There exist two positive constants $C$ and $M$, such that

$$
\begin{aligned}
& \left|D_{\sigma}(z) \Gamma_{z}(\cdot, \zeta)-D_{\sigma}\left(z_{0}\right) \Gamma_{z_{0}}(\cdot, \zeta)\right| \\
& \leq C\left(d_{z_{0}}\left(z_{0}, z\right) d_{z_{0}}\left(z_{0}, \zeta\right)^{-Q+1-|\sigma|}+d_{z_{0}}\left(z_{0}, z\right)^{\alpha} d_{z_{0}}\left(z_{0}, \zeta\right)^{-Q+2-|\sigma|}\right),
\end{aligned}
$$

for every multi-index $\sigma,|\sigma|=k$, and for every $z, z_{0} \in K, \zeta \in \Omega_{M}$ defined in (2.33).

The proof of the above statement relies on the following.

Lemma 2.22. Let $\Omega$ be a bounded open set, $\bar{u} \in C_{\bar{u}}^{1, \alpha}(\Omega), K$ a compact subset of $\Omega$ and $M>0$. Then there exists a positive constant $M_{0}$ such that $\left|\left(\theta_{z_{0}}^{\left(z_{0}\right)}(\zeta) \oplus\left(-\theta_{z}^{(z)}(\zeta)\right)\right)_{3}\right| \leq M_{0}\left(d_{z_{0}}\left(z_{0}, \zeta\right)^{3} d_{z_{0}}\left(z_{0}, z\right)^{\alpha}+d_{z_{0}}\left(z_{0}, \zeta\right)^{2} d_{z_{0}}\left(z_{0}, z\right)\right)$,

for every $z, z_{0} \in K$ and for every $\zeta \in \Omega_{M}$ (the notation $(\cdot)_{3}$ in the left-hand side denotes the third component of the considered vector). Moreover, if $M$ is sufficiently big, there exist two constants $M_{1}, M_{2}>1$ such that, for every $z, z_{0} \in K$ and for every $\zeta \in \Omega_{M}$, we have

$$
M_{1}\left\|\theta_{z_{0}}^{\left(z_{0}\right)}(\zeta) \oplus\left(-\theta_{z}^{(z)}(\zeta)\right)\right\|_{H} \leq\left\|\theta_{z_{0}}^{\left(z_{0}\right)}(\zeta)\right\|_{H}
$$

and, for every $\theta \in \mathbb{R}^{3}$ such that $d_{H}\left(\theta_{z_{0}}^{\left(z_{0}\right)}(\zeta), \theta\right) \leq d_{H}\left(\theta_{z}^{(z)}(\zeta), \theta_{z_{0}}^{\left(z_{0}\right)}(\zeta)\right)$, we have

$$
d_{z_{0}}\left(z_{0}, \zeta\right) \leq M_{2}\|\theta\|_{H} .
$$

Proof. A straightforward computation gives

$$
\begin{aligned}
\theta_{z_{0}}^{\left(z_{0}\right)}(\zeta) & \oplus\left(-\theta_{z}^{(z)}(\zeta)\right)=\left(x-x_{0}, t_{0}-t, \frac{1}{2}\left(x-x_{0}\right)\left(2 \tau-t-t_{0}\right)\right. \\
& \left.+\frac{1}{\bar{u}_{x}\left(z_{0}\right)}\left(\eta-y_{0}+\bar{u}\left(z_{0}\right)\left(\tau-t_{0}\right)\right)-\frac{1}{\bar{u}_{x}(z)}(\eta-y+\bar{u}(z)(\tau-t))\right)
\end{aligned}
$$

If we denote by $\theta_{3}$ the last component of $\theta_{z_{0}}^{\left(z_{0}\right)}(\zeta) \oplus\left(-\theta_{z}^{(z)}(\zeta)\right)$, we have

$$
\left|\theta_{3}\right| \leq \frac{1}{2}\left|x-x_{0}\right|\left(\left|\tau-t_{0}\right|+|\tau-t|\right)
$$




$$
\begin{aligned}
& \quad+\left|\left(\frac{1}{\bar{u}_{x}(z)}-\frac{1}{\bar{u}_{x}\left(z_{0}\right)}\right)\left(\eta-y_{0}+\bar{u}\left(z_{0}\right)\left(\tau-t_{0}\right)\right)\right| \\
& \quad+\left|\frac{1}{\bar{u}_{x}(z)}\left(y-y_{0}+\bar{u}\left(z_{0}\right)\left(\tau-t_{0}\right)-\bar{u}(z)(\tau-t)\right)\right| \\
& \leq C_{1}\left(\left|x-x_{0}\right|\left(\left|\tau-t_{0}\right|+|\tau-t|\right)\right. \\
& +\left|\frac{\bar{u}_{x}(z)-\bar{u}_{x}\left(z_{0}\right)}{\bar{u}_{x}(z) \bar{u}_{x}\left(z_{0}\right)}\right|\left|\eta-y_{0}+\bar{u}\left(z_{0}\right)\left(\tau-t_{0}\right)\right| \\
& +\left|y-y_{0}+\bar{u}\left(z_{0}\right)\left(t-t_{0}\right)\right|+|t-\tau|\left|\bar{u}(z)-\bar{u}\left(z_{0}\right)-\bar{u}_{x}\left(z_{0}\right)\left(x-x_{0}\right)\right| \\
& \left.+\left|\bar{u}_{x}\left(z_{0}\right)\right||t-\tau|\left|x-x_{0}\right|\right) \\
& \leq C_{2}\left(d_{z_{0}}\left(z_{0}, z\right)\left(d_{z_{0}}\left(z_{0}, \zeta\right)^{2}+d_{z_{0}}(z, \zeta)^{2}\right)+d_{z_{0}}\left(z_{0}, z\right)^{\alpha} d_{z_{0}}\left(z_{0}, \zeta\right)^{3}\right. \\
& \left.+d_{z_{0}}\left(z_{0}, z\right)^{3}+d_{z_{0}}(z, \zeta)^{2} d_{z_{0}}\left(z_{0}, z\right)^{1+\alpha}+d_{z_{0}}(z, \zeta)^{2} d_{z_{0}}\left(z_{0}, z\right)\right),
\end{aligned}
$$

since $\bar{u}_{x} \in C_{\bar{u}}^{\alpha}(\Omega)$. By using the inequality

$$
d_{z_{0}}(z, \zeta) \leq \widetilde{C}\left(d_{z_{0}}\left(z_{0}, z\right)+d_{z_{0}}\left(z_{0}, \zeta\right)\right)
$$

we then obtain

$$
\begin{aligned}
\left|\theta_{3}\right| & \leq C_{3}\left(d_{z_{0}}\left(z_{0}, \zeta\right)^{2} d_{z_{0}}\left(z_{0}, z\right)\right)+d_{z_{0}}\left(z_{0}, \zeta\right)^{3} d_{z_{0}}\left(z_{0}, z\right)^{\alpha}+d_{z_{0}}\left(z_{0}, z\right)^{3} \\
& \left.+d_{z_{0}}\left(z_{0}, \zeta\right)^{3+\alpha}+d_{z_{0}}\left(z_{0}, \zeta\right)^{2} d_{z_{0}}\left(z_{0}, z\right)^{1+\alpha}\right) .
\end{aligned}
$$

Since $z_{0}, z \in K$ and $\zeta \in \Omega_{M}$, from the above inequality we immediately deduce (2.38). Moreover, using the fact that $\Omega$ is bounded and that $\zeta \in \Omega_{M}$, we get

$$
\begin{aligned}
\left\|\theta_{z_{0}}^{\left(z_{0}\right)}(\zeta) \oplus\left(-\theta_{z}^{(z)}(\zeta)\right)\right\|_{H} & \leq d_{z_{0}}\left(z_{0}, z\right)+\left|\theta_{3}\right|^{\frac{1}{3}} \\
& \leq \frac{d_{z_{0}}\left(z_{0}, \zeta\right)}{M}+M_{0}^{\frac{1}{3}}\left(\frac{d_{z_{0}}\left(z_{0}, \zeta\right)^{3}}{M^{\alpha}}+\frac{d_{z_{0}}\left(z_{0}, \zeta\right)^{3}}{M}\right)^{\frac{1}{3}}
\end{aligned}
$$

then, by choosing $M$ sufficiently great, we also obtain (2.39). Finally, if $d_{H}\left(\theta_{z_{0}}^{\left(z_{0}\right)}(\zeta), \theta\right) \leq d_{H}\left(\theta_{z}^{(z)}(\zeta), \theta_{z_{0}}^{\left(z_{0}\right)}(\zeta)\right)$, from (2.39) we obtain

$$
\begin{aligned}
\|\theta\|_{H} & \geq d_{H}\left(\theta_{z_{0}}^{\left(z_{0}\right)}(\zeta), 0\right)-d_{H}\left(\theta_{z_{0}}^{\left(z_{0}\right)}(\zeta), \theta\right) \\
& \geq d_{H}\left(\theta_{z_{0}}^{\left(z_{0}\right)}(\zeta), 0\right)-d_{H}\left(\theta_{z}^{(z)}(\zeta), \theta_{z_{0}}^{\left(z_{0}\right)}(\zeta)\right) \\
& \geq\left(1-\frac{1}{M_{1}}\right) d_{H}\left(\theta_{z_{0}}^{\left(z_{0}\right)}(\zeta), 0\right) \geq \frac{1}{M_{2}} d_{z_{0}}\left(z_{0}, \zeta\right) .
\end{aligned}
$$

This proves (2.40) and concludes the proof of the lemma. 
Proof of Proposition 2.21. Let us first prove that, for every multi-index $\sigma,|\sigma| \geq 0$, we have

$$
\begin{aligned}
& \left|D_{\sigma}^{H} \Gamma_{H}\left(-\theta_{z}^{(z)}(\zeta)\right)-D_{\sigma}^{H} \Gamma_{H}\left(-\theta_{z_{0}}^{\left(z_{0}\right)}(\zeta)\right)\right| \\
& \leq C\left(d_{z_{0}}\left(z_{0}, z\right)^{\alpha} d_{z_{0}}\left(z_{0}, \zeta\right)^{-Q+2-|\sigma|}+d_{z_{0}}\left(z_{0}, z\right) d_{z_{0}}\left(z_{0}, \zeta\right)^{-Q+1-|\sigma|}\right),
\end{aligned}
$$

for some positive constant $C$. Indeed, we have

$$
\left|D_{\sigma}^{H} \Gamma_{H}\left(-\theta_{z}^{(z)}(\zeta)\right)-D_{\sigma}^{H} \Gamma_{H}\left(-\theta_{z_{0}}^{\left(z_{0}\right)}(\zeta)\right)\right|=
$$

(by the mean value theorem and denoting $\nabla_{H}=\left(X_{H}, Y_{H}, \partial_{\theta_{3}}\right)$ )

$$
=\left|\left\langle\theta_{z_{0}}^{\left(z_{0}\right)}(\zeta) \oplus\left(-\theta_{z}^{(z)}(\zeta)\right), \nabla_{H} D_{\sigma}^{H} \Gamma_{H}(\theta)\right\rangle\right| \leq
$$

(where $d_{H}\left(\theta_{z_{0}}^{\left(z_{0}\right)}(\zeta), \theta\right) \leq d_{H}\left(\theta_{z}^{(z)}(\zeta), \theta_{z_{0}}^{\left(z_{0}\right)}(\zeta)\right)$ )

$$
\begin{aligned}
& \leq\left|x-x_{0}\right|\left|X_{H} D_{\sigma}^{H} \Gamma_{H}(\theta)\right|+\left|t-t_{0}\right|\left|Y_{H} D_{\sigma}^{H} \Gamma_{H}(\theta)\right| \\
& +\left|\left(\theta_{z_{0}}^{\left(z_{0}\right)}(\zeta) \oplus\left(-\theta_{z}^{(z)}(\zeta)\right)\right)_{3}\right|\left|\partial_{\theta_{3}} D_{\sigma}^{H} \Gamma_{H}(\theta)\right| \leq
\end{aligned}
$$

(by $(2.40)$ and $(2.38)$ )

$$
\leq C\left(d_{z_{0}}\left(z_{0}, z\right) d_{z_{0}}\left(z_{0}, \zeta\right)^{-Q+1-|\sigma|}+d_{z_{0}}\left(z_{0}, z\right)^{\alpha} d_{z_{0}}\left(z_{0}, \zeta\right)^{-Q+2-|\sigma|}\right) .
$$

Assertion (2.41) is then proved. Now we can apply Lemma 2.17, again denoting $\widetilde{I}_{\sigma}=\left\{\varrho: k_{\varrho}=0\right\}$. We have

$$
\begin{aligned}
& \left|D_{\sigma}\left(z_{0}\right) \Gamma_{z_{0}}(\cdot, \zeta)-D_{\sigma}(z) \Gamma_{z}(\cdot, \zeta)\right| \\
& =\mid \sum_{\varrho \in \widetilde{I}_{\sigma}}\left(\frac{C_{\varrho}}{\bar{u}_{x}\left(z_{0}\right)^{h_{\varrho}}} \prod_{\mu \in I_{\varrho}} D_{\mu} \bar{u}\left(z_{0}\right) D_{\varrho}^{\left(z_{0}\right)}\left(z_{0}\right) \Gamma_{z_{0}}(\cdot, \zeta)\right. \\
& \left.-\frac{C_{\varrho}}{\bar{u}_{x}(z)^{h_{\varrho}}} \prod_{\mu \in I_{\varrho}} D_{\mu} \bar{u}(z) D_{\varrho}^{(z)}(z) \Gamma_{z_{0}}(\cdot, \zeta)\right) \mid \\
& \leq \sum_{\varrho \in \widetilde{I}_{\sigma}} C_{\varrho}\left|\frac{1}{\bar{u}_{x}\left(z_{0}\right)^{h_{\varrho}}} \prod_{\mu \in I_{\varrho}} D_{\mu} \bar{u}\left(z_{0}\right)-\frac{1}{\bar{u}_{x}(z)^{h_{\varrho}}} \prod_{\mu \in I_{\varrho}} D_{\mu} \bar{u}(z)\right|\left|D_{\varrho}^{\left(z_{0}\right)}\left(z_{0}\right) \Gamma_{z_{0}}(\cdot, \zeta)\right| \\
& +\sum_{\varrho \in \widetilde{I}_{\sigma}} C_{\varrho}\left|\prod_{\mu \in I_{\varrho}} D_{\mu} \bar{u}(z)\right|\left|D_{\varrho}^{(z)}(z) \Gamma_{z}(\cdot, \zeta)-D_{\varrho}^{\left(z_{0}\right)}\left(z_{0}\right) \Gamma_{z_{0}}(\cdot, \zeta)\right|
\end{aligned}
$$

(by $(2.29)$ )

$$
=\sum_{\varrho \in \widetilde{I}_{\sigma}} C_{\varrho}\left|\frac{1}{\bar{u}_{x}\left(z_{0}\right)^{h_{\varrho}}} \prod_{\mu \in I_{\varrho}} D_{\mu} \bar{u}\left(z_{0}\right)-\frac{1}{\bar{u}_{x}(z)^{h_{\varrho}}} \prod_{\mu \in I_{\varrho}} D_{\mu} \bar{u}(z)\right|\left|D_{\varrho}^{\left(z_{0}\right)}\left(z_{0}\right) \Gamma_{z_{0}}(\cdot, \zeta)\right|
$$




$$
+\sum_{\varrho \in \widetilde{I}_{\sigma}} C_{\varrho}\left|\prod_{\mu \in I_{\varrho}} D_{\mu} \bar{u}(z)\right|\left|\frac{1}{\bar{u}_{x}\left(z_{0}\right)}\left(D_{\varrho}^{H} \Gamma_{H}\right)\left(-\theta_{z_{0}}^{\left(z_{0}\right)}(\zeta)\right)-\frac{1}{\bar{u}_{x}(z)}\left(D_{\varrho}^{H} \Gamma_{H}\right)\left(-\theta_{z}^{(z)}(\zeta)\right)\right|
$$

(by 2.41)

$$
\leq C \sum_{\varrho \in \widetilde{I}_{\sigma}}\left(d_{z_{0}}\left(z_{0}, z\right) d_{z_{0}}\left(z_{0}, \zeta\right)^{-Q-|\varrho|+1}+d_{z_{0}}\left(z_{0}, z\right)^{\alpha} d_{z_{0}}\left(z_{0}, \zeta\right)^{-Q-|\varrho|+2}\right)
$$

(since $|\varrho| \leq|\sigma|, \forall \varrho \in I_{\varrho}$ )

$$
\leq C\left(d_{z_{0}}\left(z_{0}, z\right) d_{z_{0}}\left(z_{0}, \zeta\right)^{-Q-|\sigma|+1}+d_{z_{0}}\left(z_{0}, z\right)^{\alpha} d_{z_{0}}\left(z_{0}, \zeta\right)^{-Q-|\sigma|+2}\right) .
$$

Proposition 2.23. Let $\varphi \in C^{\infty}(\Omega)$. Then

$$
D_{\sigma}^{\left(z_{0}\right)}(z)\left(\varphi\left(\cdot \circ \zeta^{-1}\right)\right)=\sum_{\varrho \in J_{\sigma}} p_{\varrho}(\zeta)\left(D_{\varrho}^{\left(z_{0}\right)} \varphi\right)\left(z \circ \zeta^{-1}\right)
$$

where o denotes the law group (2.5) in $G_{z_{0}}$ and $p_{\varrho}$ is a polynomial of $\delta_{\lambda}^{H}$ degree $k_{\varrho}$ in the first two components of $\zeta$ such that $|\varrho| \leq k_{\varrho}+|\sigma|, \forall \varrho \in J_{\sigma}$.

Proof. If we prove the assertion on the Heisenberg group, it will be proved in any group $\left(G_{z_{0}}, \circ\right)$, by the canonical change of variables. Let us start with $|\sigma|=1$.

$$
\begin{aligned}
& X_{H}(\theta) \varphi\left(\cdot \oplus \widetilde{\theta}^{-1}\right)=\left(\partial_{\theta_{1}}-\frac{\theta_{2}}{2} \partial_{\theta_{3}}\right) \varphi\left(\theta_{1}-\widetilde{\theta}_{1}, \theta_{2}-\widetilde{\theta}_{2}, \theta_{3}-\widetilde{\theta}_{3}-\frac{1}{2}\left(\theta_{1} \widetilde{\theta}_{2}-\theta_{2} \widetilde{\theta}_{1}\right)\right) \\
& =\left(\partial_{1} \varphi-\frac{\widetilde{\theta}_{2}}{2} \partial_{3} \varphi-\frac{\theta_{2}}{2} \partial_{3} \varphi\right)\left(\theta \circ \widetilde{\theta}^{-1}\right)=\left(\partial_{1} \varphi-\frac{\theta_{2}-\widetilde{\theta}_{2}}{2} \partial_{3} \varphi-\widetilde{\theta}_{2} \partial_{3} \varphi\right)\left(\theta \circ \widetilde{\theta}^{-1}\right) \\
& =\left(X_{H} \varphi\right)\left(\theta \circ \widetilde{\theta}^{-1}\right)-\widetilde{\theta}_{2} \partial_{3} \varphi\left(\theta \circ \widetilde{\theta}^{-1}\right) .
\end{aligned}
$$

This proves the claim since the $\delta_{\lambda}^{H}$-degree of $\widetilde{\theta}_{2}$ is two. An analogous direct computation shows that

$$
Y_{H}(\theta)\left(\varphi\left(\cdot \circ \widetilde{\theta}^{-1}\right)\right)=\left(Y_{H} \varphi\right)\left(\theta \circ \widetilde{\theta}^{-1}\right)+\widetilde{\theta}_{1} \partial_{3} \varphi\left(\theta \circ \widetilde{\theta}^{-1}\right) .
$$

The general assertion follows by iterating the previous arguments.

In the sequel we shall need the following results.

Remark 2.24. If $u \in C_{\bar{u}}^{k, \alpha}(\Omega)$, the coefficients $c_{i, j, m}$ in $P_{z_{0}}^{k} u$ depend on the derivatives of $u$ of order less than or equal to $k$. Hence $c_{i, j, m} \in C_{\bar{u}}^{\alpha}(\Omega)$. If $K$ is a compact subset of $\Omega$ and $\sigma$ is a multi-index, then there exists $C>0$ such that

and

$$
\left|D_{\sigma} P_{z}^{k} u(\zeta)-D_{\sigma} P_{z_{0}}^{k} u(\zeta)\right| \leq C d_{z_{0}}\left(z_{0}, z\right)^{\alpha}
$$

$$
\left|\left(P_{z_{0}}^{k} u(\zeta)-P_{z_{0}}^{1} u(\zeta)\right)-\left(P_{z}^{k} u(\zeta)-P_{z}^{1} u(\zeta)\right)\right| \leq C d_{z_{0}}\left(z_{0}, z\right)^{\alpha} d_{z_{0}}\left(z_{0}, \zeta\right)^{2},
$$


for every $z, z_{0}, \zeta \in K$.

Remark 2.25. Let $u \in C_{\bar{u}}^{k, \alpha}(\Omega)$, with $0 \leq k \leq 5$. Then

$$
\left|P_{z_{0}}^{k} u(\zeta)-P_{z}^{k} u(\zeta)\right| \leq C d_{z_{0}}\left(z_{0}, z\right)^{\alpha} d_{z_{0}}\left(z_{0}, \zeta\right)^{k},
$$

for every $z, z_{0} \in \Omega$ and $\zeta \in \Omega_{M}$, where $\Omega_{M}$ is defined in (2.33).

\section{Regularization Results}

In this section we prove Theorems 1.1 and 1.2. We consider the linear equation in $\Omega \subseteq \mathbb{R}^{3}$,

$$
L_{\bar{u}} u=u_{x x}+\bar{u} u_{y}-u_{t}=f .
$$

We say that a function $u \in C^{1}(\Omega)$ is a classical solution of (3.1), if there exists $\partial_{x x} u \in C(\Omega)$ and equation (1.1) is satisfied at every point of $\Omega$. In order to study the regularity of $u$, we first represent it in terms of the fundamental solution $\Gamma_{z_{0}}$ :

$$
(u \varphi)(z)=\int \Gamma_{z_{0}}(z, \zeta) L_{z_{0}}(u \varphi)(\zeta) d \zeta
$$

for any $C_{0}^{\infty}(\Omega)$ function $\varphi$. Then we set, as usual,

$$
U_{\varepsilon}\left(z, z_{0}\right)=\int \Gamma_{z_{0}}(z, \zeta) \chi_{z_{0}, \varepsilon}(z, \zeta) L_{z_{0}}(u \varphi)(\zeta) d \zeta
$$

where $\chi_{z_{0}, \varepsilon}(z, \cdot)$ is a cut-off function, vanishing in a neighborhood of the pole of $\Gamma_{z_{0}}(z, \cdot)$.

As we pointed out in the introduction we can not use the standard theory, based on uniform convergence of $U_{\varepsilon}\left(z, z_{0}\right)$ and its derivatives to $u$ and its derivatives. Instead we use a different technique, introduced in [6] and [7] and based on a weak definition of local uniform convergence and on the representation of higher-order derivatives as limits of suitable different quotients.

We represent the functions $u$ and $U_{\varepsilon}$ in (3.2) and (3.3) as the sum of two terms

$$
u=I_{1}\left(\cdot, z_{0}\right)+I_{2}\left(\cdot, z_{0}\right) \quad U_{\varepsilon}=I_{1, \varepsilon}\left(\cdot, z_{0}\right)+I_{2, \varepsilon}\left(\cdot, z_{0}\right),
$$

where $I_{1}\left(\cdot, z_{0}\right)$ is $C^{\infty}, I_{1, \varepsilon}$ uniformly converges to $I_{1}$, while $I_{2, \varepsilon}$ converges to $I_{2}$ in the sense of the following definition.

Definition 3.1. Let $\left(F_{\varepsilon}\right)$ be a family of continuous functions on $\Omega \times \Omega$, let $f: \Omega \rightarrow \mathbb{R}$, let $\alpha \in(0,1)$ and $k \in \mathbb{N}$. We say that $F_{\varepsilon}\left(z, z_{0}\right) \longrightarrow f\left(z_{0}\right)$, as $\varepsilon \rightarrow 0$, locally uniformly of order $k+\alpha$ if for every compact set $K \subset \Omega$ there exists $C>0$ such that

$$
\left|F_{\varepsilon}\left(z, z_{0}\right)-f\left(z_{0}\right)\right| \leq C \varepsilon^{k+\alpha}, \quad \forall z, z_{0} \in K, d_{z_{0}}\left(z, z_{0}\right) \leq \varepsilon .
$$


We next state an existence result for the derivatives $D_{\sigma} u$ (introduced in formula $(2.31))$.

Lemma 3.2. Let $|\sigma| \geq 1$ and let $u \in C_{\bar{u}}^{|\sigma|-1, \alpha}(\Omega)$, a function which can be represented as $u(z)=I_{1}\left(z, z_{0}\right)+I_{2}\left(z, z_{0}\right)$, where $I_{1}$ is smooth as a function of $z$ in $\Omega$, and the function $z \rightarrow D_{\sigma}(z) I_{1}\left(\cdot, z_{0}\right)$ is continuous in $z$ uniformly in $z_{0}$. Assume that there exists a family $I_{2, \varepsilon}$ of smooth functions and a continuous function $I_{2}^{\sigma}$ such that $I_{2, \varepsilon}\left(z, z_{0}\right) \longrightarrow I_{2}\left(z_{0}, z_{0}\right)$, as $\varepsilon \rightarrow 0$, locally uniformly of order $|\sigma|+\alpha$, and $D_{\sigma} I_{2, \varepsilon}\left(z, z_{0}\right) \longrightarrow I_{2}^{\sigma}\left(z_{0}\right)$, as $\varepsilon \rightarrow 0$, locally uniformly of order $\alpha$. Then $D_{\sigma} u\left(z_{0}\right)$ exists and, for every $z_{0}$ in $\Omega$,

$$
D_{\sigma} u\left(z_{0}\right)=D_{\sigma}\left(z_{0}\right) I_{1}\left(\cdot, z_{0}\right)+I_{2}^{\sigma}\left(z_{0}\right) \text {. }
$$

The proof is postponed to Subsection 3.1. In Subsection 3.2, we prove Theorem 1.2 by using Lemma 3.2. Finally, in Subsection 3.3, we conclude the proof of Theorem 1.1.

3.1. Derivatives and difference quotients. The main ideas of the proof of Lemma 3.2 are already contained in [6] and [7], but the lemma is not stated explicitly; hence we give here the proof. It is based on the following definition:

Definition 3.3. If $g: \Omega \longrightarrow \mathbb{R}$, for every $z \in \Omega$ and $h \in \mathbb{R}$ sufficiently small, we define

$$
\triangle_{(i)}(z) g(h)=\frac{g\left(\exp \left(h^{i} D_{(i)}\right)(z)\right)-g(z)}{h^{i}}, \quad i=1,2 .
$$

For every multi-index $\sigma=\left(\sigma_{1}, \ldots, \sigma_{m}\right) \in\{1,2\}^{m}$, we define by recurrence

$$
\triangle_{\sigma}(z) g(h)=\triangle_{\left(\sigma_{1}\right)}(z)\left(\triangle_{\left(\sigma_{2}, \ldots, \sigma_{m}\right)} g(h)\right)(h) .
$$

Remark 3.4. If $g \in C_{\bar{u}}^{|\sigma|}(\Omega)$ then, by the mean value theorem, we have

$$
\triangle_{\sigma}(z) g(h)=D_{\sigma} g\left(z_{h}\right),
$$

for a suitable $z_{h}$ such that $d_{\bar{u}}\left(z_{h}, z\right) \leq h$. Hence there exists

$$
\lim _{h \rightarrow 0} \triangle_{\sigma}(z) g(h)=D_{\sigma} g(z),
$$

uniformly on the compact sets.

As in [7], Remark 4.2, the following result holds.

Lemma 3.5. Let $|\sigma| \geq 1$ and let $g \in C_{\bar{u}}^{|\sigma|-1}(\Omega)$. If there exists

$$
\lim _{h \rightarrow 0} \triangle_{\sigma} g(h)=w
$$

uniformly on the compact subsets of $\Omega$, then there exists $D_{\sigma} g=w$. 
Proof of Lemma 3.2 Since $z \mapsto I_{1}\left(z, z_{0}\right)$ is smooth, by Remark 3.4, we have

$$
\Delta_{\sigma}(h) I_{1}\left(\cdot, z_{0}\right)\left(z_{0}\right) \longrightarrow D_{\sigma}\left(z_{0}\right) I_{1}\left(\cdot, z_{0}\right),
$$

as $h \rightarrow 0$, locally uniformly on compact sets. On the other side

$$
\begin{aligned}
& \left|\Delta_{\sigma}\left(z_{0}\right) I_{2}\left(\cdot, z_{0}\right)(h)-I_{2}^{\sigma}\left(z_{0}\right)\right| \\
\leq & \left|\Delta_{\sigma}\left(z_{0}\right) I_{2}\left(\cdot, z_{0}\right)(h)-\Delta_{\sigma}\left(z_{0}\right) I_{2, \varepsilon}\left(\cdot, z_{0}\right)(h)\right|+\left|\Delta_{\sigma}\left(z_{0}\right) I_{2, \varepsilon}\left(\cdot, z_{0}\right)(h)-I_{2}^{\sigma}\left(z_{0}\right)\right|
\end{aligned}
$$

(by the hypotheses on the local uniform convergence of order $k+\alpha$ and Remark 3.4)

$$
\leq C_{1} \varepsilon^{\alpha}+\left|D_{\sigma}\left(z_{h}\right) I_{2, \varepsilon}\left(\cdot, z_{0}\right)-I_{2}^{\sigma}\left(z_{0}\right)\right| \leq C \varepsilon^{\alpha}
$$

Then

$$
\Delta_{\sigma}\left(z_{0}\right) u(h) \longrightarrow D_{\sigma}\left(z_{0}\right) I_{1}\left(\cdot, z_{0}\right)+I_{2}^{\sigma}\left(z_{0}\right),
$$

as $h \rightarrow 0$, uniformly on the compact sets. By Lemma 3.5, we infer that

$$
D_{\sigma}\left(z_{0}\right) u=D_{\sigma}\left(z_{0}\right) I_{1}\left(\cdot, z_{0}\right)+I_{2}^{\sigma}\left(z_{0}\right) .
$$

3.2. Linear operators with $C_{\bar{u}}^{k, \alpha}$ coefficients. The aim of this subsection is the proof of Theorem 1.2. Let $K$ be fixed according to Remark 2.5 and let $K_{1}$ be any compact set $K_{1} \subset \subset \operatorname{int}(K)$. We study the regularity of $u$ in $K_{1}$. We fix a function $\varphi \in C_{0}^{\infty}(\operatorname{int}(K))$ such that $\varphi \equiv 1$ in a neighborhood of $K_{1}$. It is nonrestrictive to assume that, if $z, z_{0} \in K_{1}$, then

$$
M d_{z_{0}}\left(z_{0}, z\right) \leq d_{z_{0}}\left(K_{1}, \operatorname{supp}(\nabla \varphi)\right), \quad M d_{z}\left(z, z_{0}\right) \leq d_{z}\left(K_{1}, \operatorname{supp}(\nabla \varphi)\right),
$$

where $M$ is the constant of Lemma 2.22 .

Remark 3.6. With this choice of function $\varphi$ and compact set $K_{1}$, we have

$$
C_{1} d_{z}(z, \zeta) \leq d_{z_{0}}\left(z_{0}, \zeta\right) \leq C_{2} d_{z}(z, \zeta), \quad \forall \zeta \in \operatorname{supp}(\nabla \varphi), z, z_{0} \in K_{1}
$$

where $C_{1}, C_{2}$ are positive constants depending only on $\bar{u}$ and $K$. In particular,

$$
d_{z_{0}}\left(z_{0}, z\right) \leq C d_{z_{0}}(z, \zeta), \quad \forall \zeta \in \operatorname{supp}(\nabla \varphi), z, z_{0} \in K
$$

Proof. By (3.4) and Proposition 2.4-(ii), we have

$$
d_{z_{0}}\left(z_{0}, \zeta\right) \leq C\left(d_{z_{0}}\left(z_{0}, z\right)+d_{z}(z, \zeta)\right) \leq C\left(\frac{1}{M} d_{z_{0}}\left(z_{0}, \zeta\right)+d_{z}(z, \zeta)\right),
$$

$\forall \zeta \in \operatorname{supp}(\nabla \varphi)$; thus, if $M$ is sufficiently large, we get

$$
d_{z_{0}}\left(z_{0}, \zeta\right) \leq C d_{z}(z, \zeta)
$$

for every $\zeta \in \operatorname{supp}(\nabla \varphi)$. Exchanging the role of $z_{0}$ and $z$ in (3.7), we get (3.5). 
Proposition 3.7. Let us assume that the coefficient $\bar{u}$ in equation (3.1) is of class $C_{\bar{u}}^{k-1, \alpha}(\Omega), 2 \leq k \leq 6$, and that $f$ is of class $C_{\bar{u}}^{k-2, \alpha}(\Omega)$. Let $u \in C_{\bar{u}}^{k-1, \alpha}(\Omega)$ be a classical solution of (3.1). Then, for every $z, z_{0} \in K_{1}$, $u=u \varphi$ can be represented as

$$
\begin{aligned}
u(z)=u \varphi(z) & =\int_{\Omega} \Gamma_{z_{0}}(z, \zeta) N_{1}\left(\zeta, z_{0}\right) d \zeta \\
& +\int_{\Omega} \Gamma_{z_{0}}(z, \zeta) N_{2, k}\left(\zeta, z_{0}\right) d \zeta+\int_{\Omega} \Gamma_{z_{0}}(z, \zeta) N_{3, k}\left(\zeta, z_{0}\right) d \zeta .
\end{aligned}
$$

where $N_{i, k}\left(\cdot, z_{0}\right)$ is supported in the support of $\varphi$, and

(i) $\operatorname{supp}\left(N_{1}\left(\cdot, z_{0}\right)\right) \subseteq \operatorname{supp}(\nabla \varphi)$ and

$$
\left|N_{1}\left(\zeta, z_{0}\right)-N_{1}(\zeta, z)\right| \leq C d_{z_{0}}\left(z_{0}, z\right)^{\alpha} d_{z_{0}}\left(z_{0}, \zeta\right)
$$

(ii) $N_{2, k}\left(\cdot, z_{0}\right)$ is of class $C^{\infty}$ and for every multi-index $\sigma$

$$
\left|D_{\sigma}(\zeta) N_{2, k}\left(\cdot, z_{0}\right)-D_{\sigma}(\zeta) N_{2, k}(\cdot, z)\right| \leq C d_{z_{0}}\left(z_{0}, z\right)^{\alpha}, \quad \forall \zeta \in K ;
$$

(iii) there exists a constant $C$, dependent only on the choice of $\varphi$ and $K_{1}$, such that for every $\zeta \in K$ and $z, z_{0} \in K_{1}$

$$
\begin{aligned}
\left|N_{3, k}\left(\zeta, z_{0}\right)\right| & \leq C d_{z_{0}}^{k-2+\alpha}\left(z_{0}, \zeta\right), \\
\left|N_{3, k}\left(\zeta, z_{0}\right)-N_{3, k}(\zeta, z)\right| & \leq C d_{z_{0}}\left(z_{0}, z\right)^{\alpha} d_{z_{0}}\left(z_{0}, \zeta\right)^{k-2} .
\end{aligned}
$$

Proof. By definition of the fundamental solution, we have

$$
\begin{aligned}
& u \varphi(z)=\int_{\Omega} \Gamma_{z_{0}}(z, \zeta) L_{z_{0}}(u \varphi)(\zeta) d \zeta=\int_{\Omega} \Gamma_{z_{0}}(z, \zeta)\left(u L_{z_{0}} \varphi+2 X u X \varphi\right) d \zeta \\
& +\int_{\Omega} \Gamma_{z_{0}}(z, \zeta) L_{\bar{u}} u(\zeta) \varphi(\zeta) d \zeta+\int_{\Omega} \Gamma_{z_{0}}(z, \zeta)\left(L_{z_{0}}-L_{\bar{u}}\right) u(\zeta) \varphi(\zeta) d \zeta \\
& =\int_{\Omega} \Gamma_{z_{0}}(z, \zeta)\left(u L_{z_{0}} \varphi+2 X u X \varphi\right) d \zeta \\
& +\int_{\Omega} \Gamma_{z_{0}}(z, \zeta) f(\zeta) \varphi(\zeta) d \zeta-\int_{\Omega} \Gamma_{z_{0}}(z, \zeta)\left(\bar{u}-P_{z_{0}}^{1} \bar{u}\right)(\zeta) \partial_{\eta} u(\zeta) \varphi(\zeta) d \zeta
\end{aligned}
$$

In order to use a uniform notation, in the sequel we will set $P_{z_{0}}^{k} u=0$ for $k$ a negative integer. Then, for every $k \geq 2$, we have

$$
\begin{gathered}
\left(\bar{u}-P_{z_{0}}^{1} \bar{u}\right)(\zeta) \partial_{\eta} u(\zeta)=\left(P_{z_{0}}^{k-1} \bar{u}(\zeta)-P_{z_{0}}^{1} \bar{u}(\zeta)\right)\left(u_{\eta}(\zeta)-P_{z_{0}}^{k-4} u_{\eta}(\zeta)\right) \\
\quad+\left(P_{z_{0}}^{k-1} \bar{u}(\zeta)-P_{z_{0}}^{1} \bar{u}(\zeta)\right) P_{z_{0}}^{k-4} u_{\eta}(\zeta)+\left(\bar{u}-P_{z_{0}}^{k-1} \bar{u}\right)(\zeta) \partial_{\eta} u(\zeta) .
\end{gathered}
$$


Then (3.8) is satisfied by inserting these expressions in formula (3.12) and by choosing the kernels $N_{1}, N_{2, k}$, and $N_{3, k}$ as follows:

$$
\begin{aligned}
N_{1}\left(\zeta, z_{0}\right) & =u(\zeta) L_{z_{0}} \varphi(\zeta)+2 X u(\zeta) X \varphi(\zeta), \\
N_{2, k}\left(\zeta, z_{0}\right) & =P_{z_{0}}^{k-2} f(\zeta) \varphi(\zeta)+\left(P_{z_{0}}^{k-1} \bar{u}(\zeta)-P_{z_{0}}^{1} \bar{u}(\zeta)\right) P_{z_{0}}^{k-4} u_{\eta}(\zeta) \varphi(\zeta), \\
N_{3, k}\left(\zeta, z_{0}\right) & =\left(f(\zeta)-P_{z_{0}}^{k-2} f(\zeta)\right) \varphi(\zeta)+\left(\bar{u}(\zeta)-P_{z_{0}}^{k-1} \bar{u}(\zeta)\right) u_{\eta}(\zeta) \varphi(\zeta) \\
& +\left(P_{z_{0}}^{k-1} \bar{u}(\zeta)-P_{z_{0}}^{1} \bar{u}(\zeta)\right)\left(u_{\eta}(\zeta)-P_{z_{0}}^{k-4} u_{\eta}(\zeta)\right) \varphi(\zeta) .
\end{aligned}
$$

Let us prove (i). The support of $N_{1}\left(\cdot, z_{0}\right)$ is clearly a subset of $\operatorname{supp}(\nabla \varphi)$. Formula (3.9) can be proved as follows:

$$
\begin{aligned}
\left|N_{1}\left(\zeta, z_{0}\right)-N_{1}(\zeta, z)\right| & =\left|u(\zeta)\left(L_{z_{0}} \varphi(\zeta)-L_{z} \varphi(\zeta)\right)\right| \\
& =|u(\zeta)|\left|P_{z_{0}}^{1} \bar{u}(\zeta)-P_{z}^{1} \bar{u}(\zeta)\right|\left|\partial_{\eta} \varphi(\zeta)\right| \leq
\end{aligned}
$$

(by Remark 2.25)

$$
\leq C\left(d_{z_{0}}\left(z_{0}, z\right)^{1+\alpha}+d_{z_{0}}\left(z_{0}, z\right)^{\alpha} d_{z_{0}}\left(z_{0}, \zeta\right)\right) \leq C d_{z_{0}}\left(z_{0}, z\right)^{\alpha} d_{z_{0}}\left(z_{0}, \zeta\right)
$$

Condition (ii) easily follows from the definition of $N_{2, k}$ and Remark 2.24.

Let us prove (3.10) for $k=2$ :

$N_{3,2}\left(\zeta, z_{0}\right)=\left(f(\zeta)-f\left(z_{0}\right)\right) \varphi(\zeta)+\left(\bar{u}(\zeta)-P_{z_{0}}^{1} \bar{u}(\zeta)\right) u_{\eta}(\zeta) \varphi(\zeta) \leq C d_{z_{0}}\left(z_{0}, \zeta\right)^{\alpha}$.

We observe that, for every $k \geq 3$,

$$
P_{z_{0}}^{k-1} \bar{u}(\zeta)-P_{z_{0}}^{1} \bar{u}(\zeta)=O\left(d_{z_{0}}\left(z_{0}, \zeta\right)^{2}\right), \quad \text { as } \zeta \rightarrow z_{0}
$$

Hence, we get

$$
\begin{aligned}
N_{3,3}\left(\zeta, z_{0}\right) & =\left(f(\zeta)-P_{z_{0}}^{1} f(\zeta)\right) \varphi(\zeta)+\left(\bar{u}(\zeta)-P_{z_{0}}^{2} \bar{u}(\zeta)\right) u_{\eta}(\zeta) \varphi(\zeta) \\
& +O\left(d_{z_{0}}\left(z_{0}, \zeta\right)^{2}\right)=O\left(d_{z_{0}}\left(z_{0}, \zeta\right)^{1+\alpha}\right), \quad \text { as } \zeta \rightarrow z_{0}
\end{aligned}
$$

This proves (3.10) for $k=3$. We can proceed analogously for $k \geq 4$.

Let us prove (3.11):

$$
\begin{aligned}
& \left|N_{3, k}\left(\zeta, z_{0}\right)-N_{3, k}(\zeta, z)\right| \leq\left|P_{z_{0}}^{k-2} f(\zeta)-P_{z}^{k-2} f(\zeta)\right||\varphi(\zeta)| \\
& +\left|P_{z_{0}}^{k-1} \bar{u}(\zeta)-P_{z_{0}}^{1} \bar{u}(\zeta)\right|\left|P_{z_{0}}^{k-4} u_{\eta}(\zeta)-P_{z}^{k-4} u_{\eta}(\zeta)\right||\varphi(\zeta)| \\
& +\left|\left(P_{z_{0}}^{k-1} \bar{u}(\zeta)-P_{z_{0}}^{1} \bar{u}(\zeta)\right)-\left(P_{z}^{k-1} \bar{u}(\zeta)-P_{z}^{1} \bar{u}(\zeta)\right)\right|\left|u_{\eta}(\zeta)-P_{z_{0}}^{k-4} u_{\eta}(\zeta)\right||\varphi(\zeta)| \\
& +\left|P_{z_{0}}^{k-1} \bar{u}(\zeta)-P_{z}^{k-1} \bar{u}(\zeta)\right|\left|u_{\eta}(\zeta)\right||\varphi(\zeta)| \leq
\end{aligned}
$$

(by Remark 2.25 and Remark 2.24)

$$
\leq C d_{z_{0}}\left(z_{0}, z\right)^{\alpha} d_{z_{0}}\left(z_{0}, \zeta\right)^{k-2}
$$


Remark 3.8. As stated in formula (3.3), we introduce a cut-off function $\chi_{z_{0}, \varepsilon}(z, \zeta)$ with the following properties:

(i) $\chi_{z_{0}, \varepsilon}(\cdot, \zeta) \in C^{\infty}(\Omega,[0,1])$;

(ii) $\chi_{z_{0}, \varepsilon}(z, \zeta)=0$ if $d_{z_{0}}(z, \zeta) \leq 2 \widetilde{C} \varepsilon$;

(iii) $\chi_{z_{0}, \varepsilon}(z, \zeta)=1$ if $d_{z_{0}}(z, \zeta) \geq 4 \widetilde{C} \varepsilon$;

(iv) $\left|D_{\sigma}(z) \chi_{z_{0}, \varepsilon}(\cdot, \zeta)\right| \leq \frac{C}{\varepsilon^{|\sigma|}}$, for every multi-index $\sigma$;

for every $z_{0}, \zeta \in \Omega, \varepsilon>0$, where $\widetilde{C}$ is the constant in (2.6).

Proof. We consider a smooth function $g_{z_{0}}$ defined in terms of the composition law (2.5) of $G_{z_{0}}$. We assume that $g_{z_{0}}$ is $\delta_{\lambda}^{\left(z_{0}\right)}$-homogeneous of degree one and that

$$
\frac{3}{4} d_{z_{0}}(z, \zeta) \leq g_{z_{0}}\left(\zeta^{-1} \circ z\right) \leq \frac{5}{4} d_{z_{0}}(z, \zeta), \quad \forall z, \zeta \in \Omega .
$$

We next denote by $\chi$ a $C^{\infty}([0,+\infty),[0,1])$ function such that

$$
\chi(s)=0, \text { for } s \leq \frac{5}{4}, \quad \chi(s)=1, \text { for } s \geq \frac{3}{2},
$$

and we define $\chi_{z_{0}}(z, \zeta)=\chi\left(\frac{g_{z_{0}}\left(\zeta^{-1} \circ z\right)}{2 \widetilde{C} \varepsilon}\right)$.

Proposition 3.9. Assume that $u$ is of class $C_{\bar{u}}^{k-1, \alpha}(\Omega)$, with $2 \leq k \leq 6$, and that it can be represented as in (3.8), for every $z, z_{0} \in K$, with the kernels $N_{1}, N_{2, k}$ and $N_{3, k}$ satisfying (i), (ii) and (iii). Then $u$ is of class $C_{\bar{u}}^{k}(\Omega)$.

Proof. Since $u$ is represented as in (3.8), we apply Lemma 3.2 to it. If we set

$I_{1}\left(z, z_{0}\right)=\int_{\Omega} \Gamma_{z_{0}}(z, \zeta) N_{1}\left(\zeta, z_{0}\right) d \zeta$ and $I_{2, k}\left(z, z_{0}\right)=\int_{\Omega} \Gamma_{z_{0}}(z, \zeta) N_{2, k}\left(\zeta, z_{0}\right) d \zeta$,

then $z \mapsto I_{1}\left(z, z_{0}\right)$ is $C^{\infty}$, since $N_{1}\left(\zeta, z_{0}\right)$ is null in a neighborhood of the pole of $\Gamma_{z_{0}}(z, \zeta)$, by (i) and (3.6). Also $I_{2, k}\left(\cdot, z_{0}\right)$ is $C^{\infty}$ since, by the change of variable $\zeta^{-1} \circ z=\omega$, it can be represented as

$$
I_{2, k}\left(z, z_{0}\right)=\int \Gamma_{z_{0}}(\omega, 0) N_{2, k}\left(z \circ \omega^{-1}, z_{0}\right) d \omega,
$$

where $N_{2, k}\left(\cdot, z_{0}\right)$ is of class $C_{0}^{\infty}(\Omega)$. Next, we set

$$
I_{3, k}\left(z, z_{0}\right)=\int_{\Omega} \Gamma_{z_{0}}(z, \zeta) N_{3, k}\left(\zeta, z_{0}\right) d \zeta
$$

and, for every multi-index $\sigma$ of height $|\sigma|=k$,

$$
I_{3, k}^{\sigma}\left(z_{0}\right)=\int_{\Omega} D_{\sigma}\left(z_{0}\right) \Gamma_{z_{0}}(\cdot, \zeta) N_{3, k}\left(\zeta, z_{0}\right) d \zeta .
$$


We remark that $I_{3, k}^{\sigma}$ is well defined and continuous by (iii). Let us define

$$
I_{3, k, \varepsilon}\left(z, z_{0}\right)=\int_{\Omega} \Gamma_{z_{0}}(z, \zeta) \chi_{z_{0}, \varepsilon}(z, \zeta) N_{3, k}\left(\zeta, z_{0}\right) d \zeta .
$$

Clearly $I_{3, k, \varepsilon}\left(\cdot, z_{0}\right)$ is smooth.

In order to apply Lemma 3.2, we have only to prove that

$$
\begin{aligned}
& \sup _{d_{z_{0}}\left(z_{0}, z\right) \leq \varepsilon}\left|I_{3, k}\left(z, z_{0}\right)-I_{3, k, \varepsilon}\left(z, z_{0}\right)\right| \leq C \varepsilon^{k+\alpha}, \\
& \sup _{d_{z_{0}}\left(z_{0}, z\right) \leq \varepsilon}\left|D_{\sigma}(z) I_{3, k, \varepsilon}\left(\cdot, z_{0}\right)-I_{3, k}^{\sigma}\left(z_{0}\right)\right| \leq C \varepsilon^{\alpha},
\end{aligned}
$$

where $C$ is a positive constant which depends only on $K_{1}$. Then we will deduce that

$$
D_{\sigma} u\left(z_{0}\right)=D_{\sigma}\left(z_{0}\right) I_{1}\left(\cdot, z_{0}\right)+D_{\sigma}\left(z_{0}\right) I_{2, k}\left(\cdot, z_{0}\right)+I_{3, k}^{\sigma}\left(z_{0}\right) .
$$

Indeed, we have, by (iii),

$$
\begin{aligned}
& \left|I_{3, k}\left(z, z_{0}\right)-I_{3, k, \varepsilon}\left(z, z_{0}\right)\right| \leq C_{1} \int_{d_{z_{0}}(z, \zeta) \leq 2 \varepsilon} d_{z_{0}}(z, \zeta)^{-Q+2} d_{z_{0}}\left(z_{0}, \zeta\right)^{k-2+\alpha} d \zeta \\
\leq & C_{2} \int_{d_{z_{0}}(z, \zeta) \leq 2 \varepsilon} d_{z_{0}}(z, \zeta)^{-Q+2}\left(d_{z_{0}}\left(z_{0}, z\right)^{k-2+\alpha}+d_{z_{0}}(z, \zeta)^{k-2+\alpha}\right) d \zeta \leq C \varepsilon^{k+\alpha}
\end{aligned}
$$

since $d_{z_{0}}\left(z_{0}, z\right) \leq \varepsilon$ and (3.15) holds. We remark that

$$
D_{\sigma}(f g)=\sum_{\left|\sigma^{\prime}\right|+\left|\sigma^{\prime \prime}\right|=|\sigma|} c\left(\sigma, \sigma^{\prime}, \sigma^{\prime \prime}\right) D_{\sigma^{\prime}} f D_{\sigma^{\prime \prime}} g,
$$

for some constants $c\left(\sigma, \sigma^{\prime}, \sigma^{\prime \prime}\right)$. Thus, we have

$$
\left|D_{\sigma}(z) I_{3, k, \varepsilon}\left(\cdot, z_{0}\right)-I_{3, k}^{\sigma}\left(z_{0}\right)\right| \leq\left|B_{1}(\varepsilon)\right|+\left|B_{2}(\varepsilon)\right|+\left|B_{3}(\varepsilon)\right|,
$$

where

$$
\begin{aligned}
& B_{1}(\varepsilon)=\int_{\Omega}\left(D_{\sigma}(z)-D_{\sigma}\left(z_{0}\right)\right) \Gamma_{z_{0}}(\cdot, \zeta) \chi_{z_{0}, \varepsilon}(z, \zeta) N_{3, k}\left(\zeta, z_{0}\right) d \zeta \\
& B_{2}(\varepsilon)=\int_{\Omega} D_{\sigma}\left(z_{0}\right) \Gamma_{z_{0}}(\cdot, \zeta)\left(1-\chi_{z_{0}, \varepsilon}(z, \zeta)\right) N_{3, k}\left(\zeta, z_{0}\right) d \zeta \\
& B_{3}(\varepsilon)=\int_{\Omega} \sum_{\substack{\left|\sigma^{\prime}\right|+\left|\sigma^{\prime \prime}\right|=|\sigma|,\left|\sigma^{\prime \prime}\right| \neq 0}} c\left(\sigma, \sigma^{\prime}, \sigma^{\prime \prime}\right) D_{\sigma^{\prime}}(z) \Gamma_{z_{0}}(\cdot, \zeta) D_{\sigma^{\prime \prime}}(z) \chi_{z_{0}, \varepsilon}(z, \zeta) N_{3, k}\left(\zeta, z_{0}\right) d \zeta .
\end{aligned}
$$


Let us estimate each term separately. We observe that, for every $\zeta \in$ $\operatorname{supp}\left(\chi_{z_{0}, \varepsilon}(z, \zeta)\right)$, we have $d_{z_{0}}(z, \zeta) \geq 2 \widetilde{C} \varepsilon$, where $\widetilde{C}$ is the constant in $(2.6)$; then

$$
d_{z_{0}}\left(z_{0}, \zeta\right) \geq \frac{1}{\widetilde{C}} d_{z_{0}}(z, \zeta)-d_{z_{0}}\left(z_{0}, z\right) \geq \varepsilon
$$

Thus, by Proposition 2.20, we get

$$
\begin{aligned}
& \left|B_{1}(\varepsilon)\right| \leq \\
& C_{1} \int_{\Omega}\left(d_{z_{0}}\left(z_{0}, z\right) d_{z_{0}}\left(z_{0}, \zeta\right)^{-Q-1+\alpha}+d_{z_{0}}\left(z_{0}, z\right)^{\alpha} d_{z_{0}}\left(z_{0}, \zeta\right)^{-Q+\alpha}\right) \chi_{z_{0}, \varepsilon}(z, \zeta) d \zeta
\end{aligned}
$$

(by $(3.18)$ )

$$
\leq C_{2} \int_{d_{z_{0}}\left(z_{0}, \zeta\right) \geq \varepsilon}\left(\varepsilon d_{z_{0}}\left(z_{0}, \zeta\right)^{-Q-1+\alpha}+\varepsilon^{\alpha} d_{z_{0}}\left(z_{0}, \zeta\right)^{-Q+\alpha}\right)|\varphi(\zeta)| d \zeta \leq C \varepsilon^{\alpha} .
$$

By Remark 2.19 and since $d_{z_{0}}\left(z_{0}, \zeta\right) \leq \widetilde{C}\left(d_{z_{0}}\left(z_{0}, z\right)+d_{z_{0}}(z, \zeta)\right)$, we obtain

$$
\left|B_{2}(\varepsilon)\right| \leq C_{1} \int_{d_{z_{0}}\left(z_{0}, \zeta\right) \leq \widetilde{C}(1+4 \widetilde{C}) \varepsilon} d_{z_{0}}\left(z_{0}, \zeta\right)^{-Q+\alpha} d \zeta \leq C \varepsilon^{\alpha}
$$

Finally, by using again Remark 2.19 and property (iv) of $\chi_{z_{0}, \varepsilon}$, we obtain

$$
\begin{aligned}
\left|B_{3}(\varepsilon)\right| & \leq \sum_{\substack{\left|\sigma^{\prime}\right|+\left|\sigma^{\prime \prime}\right|=|\sigma|,\left|\sigma^{\prime \prime}\right| \neq 0}} \frac{\left|c\left(\sigma, \sigma^{\prime}, \sigma^{\prime \prime}\right)\right|}{\varepsilon^{\left|\sigma^{\prime \prime}\right|}} \int_{\varepsilon \leq d_{z_{0}}\left(z_{0}, \zeta\right) \leq \widetilde{C}(1+4 \widetilde{C}) \varepsilon} d_{z_{0}}(z, \zeta)^{-Q+2-\left|\sigma^{\prime}\right|} d_{z_{0}}\left(z_{0}, \zeta\right)^{|\sigma|-2+\alpha} d \zeta \\
& \leq C \varepsilon^{\alpha} .
\end{aligned}
$$

This concludes the proof of (3.16).

Lemma 3.10. Assume that $u$ is of class $C_{\bar{u}}^{k-1, \alpha}(\Omega)$, with $2 \leq k \leq 6, \alpha>\frac{1}{2}$, and that it can be represented as in (3.8), for every $z, z_{0} \in K$, with the kernels $N_{1}, N_{2, k}$ and $N_{3, k}$ satisfying (i), (ii) and (iii). Then

(i) $D_{\sigma} u \in C_{\bar{u}}^{\alpha^{\prime}}(\Omega)$, for every multi-index $\sigma$ of height $k$;

(ii) $D_{\sigma^{\prime}} u \in C_{Y_{\bar{u}}}^{\alpha^{\prime}}(\Omega)$, for every multi-index $\sigma^{\prime}$ of height $k-1$, for every $\alpha^{\prime} \in(0, \alpha)$.

Proof. Let us prove (i). In formula (3.17), we gave an explicit expression of $D_{\sigma} u$ as a sum of three terms. Since these terms have similar behaviour and $D_{\sigma} I_{1}$ is the simplest one, we study only $I_{3, k}^{\sigma}$ and $D_{\sigma} I_{2, k}$.

Let us start with $I_{3, k}^{\sigma}$. Let $M=M(K)$ be as in Lemma 2.22 and let $\Omega_{M}$ be the set defined in (2.33). Then, we have

$$
I_{3, k}^{\sigma}(z)-I_{3, k}^{\sigma}\left(z_{0}\right)=A_{1}\left(z, z_{0}\right)+A_{2}\left(z, z_{0}\right)+A_{3}\left(z, z_{0}\right)+A_{4}\left(z, z_{0}\right),
$$


where

$$
\begin{aligned}
& A_{1}\left(z, z_{0}\right)=\int_{\Omega_{M}}\left(D_{\sigma}(z) \Gamma_{z}(\cdot, \zeta)-D_{\sigma}\left(z_{0}\right) \Gamma_{z_{0}}(\cdot, \zeta)\right) N_{3, k}\left(\zeta, z_{0}\right) d \zeta \\
& A_{2}\left(z, z_{0}\right)=\int_{\Omega_{M}} D_{\sigma}(z) \Gamma_{z}(\cdot, \zeta)\left(N_{3, k}(\zeta, z)-N_{3, k}\left(\zeta, z_{0}\right)\right) d \zeta \\
& A_{3}\left(z, z_{0}\right)=\int_{\Omega \backslash \Omega_{M}} D_{\sigma}(z) \Gamma_{z}(\cdot, \zeta) N_{3, k}(\zeta, z) d \zeta \\
& A_{4}\left(z, z_{0}\right)=-\int_{\Omega \backslash \Omega_{M}} D_{\sigma}\left(z_{0}\right) \Gamma_{z_{0}}(\cdot, \zeta) N_{3, k}\left(\zeta, z_{0}\right) d \zeta
\end{aligned}
$$

We get immediately, by Remark 2.19,

$$
\left|A_{3}\left(z, z_{0}\right)\right|,\left|A_{4}\left(z, z_{0}\right)\right| \leq C d_{z_{0}}\left(z_{0}, z\right)^{\alpha} .
$$

Moreover, by Proposition 2.21, we have

$$
\begin{aligned}
\left|A_{1}\left(z, z_{0}\right)\right| & \leq C_{1} \int_{\Omega_{M}}\left(d_{z_{0}}\left(z_{0}, z\right) d_{z_{0}}\left(z_{0}, \zeta\right)^{-Q+\alpha-1}+d_{z_{0}}\left(z_{0}, z\right)^{\alpha} d_{z_{0}}\left(z_{0}, \zeta\right)^{-Q+\alpha}\right) d \zeta \\
& \leq C d_{z_{0}}\left(z_{0}, z\right)^{\alpha} .
\end{aligned}
$$

By Remark 2.19 and condition (iii) of Proposition 3.7, we have

$$
\left|A_{2}\left(z, z_{0}\right)\right| \leq C_{1} \int_{\Omega_{M}} d_{z}(z, \zeta)^{-Q} d_{z_{0}}\left(z_{0}, z\right)^{\alpha}|\varphi(\zeta)| d \zeta \leq
$$

(by (2.34) which holds for every $\zeta \in \Omega_{M}$, for every $\alpha^{\prime}<\alpha$ )

$$
\leq C_{2} d_{z_{0}}\left(z_{0}, z\right)^{\alpha^{\prime}} \int_{\Omega_{M}} d_{z_{0}}\left(z_{0}, \zeta\right)^{-Q+\alpha-\alpha^{\prime}}|\varphi(\zeta)| d \zeta \leq C d_{z_{0}}\left(z_{0}, z\right)^{\alpha^{\prime}} .
$$

This concludes the proof of the Hölder continuity of $I_{3, k}^{\sigma}$ of order $\alpha^{\prime}$, for every $\alpha^{\prime}<\alpha$. Let us consider $I_{2, k}$. By (3.13),

$$
I_{2, k}\left(z, z_{0}\right)=\int \Gamma_{z_{0}}(\omega) N_{2, k}\left(z \circ \omega^{-1}, z_{0}\right) d \omega,
$$

where $N_{2, k}\left(\zeta, z_{0}\right)$ is introduced in Proposition 3.7 and $\Gamma_{z_{0}}(\omega) \equiv \Gamma_{z_{0}}(\omega, 0)$. We have to show that $D_{\sigma}\left(z_{0}\right) I_{2, k}\left(\cdot, z_{0}\right) \in C_{\bar{u}}^{\alpha^{\prime}}(\Omega)$. By differentiating the above integral, we obtain

$$
D_{\sigma}\left(z_{0}\right) I_{2, k}\left(\cdot, z_{0}\right)=\int \Gamma_{z_{0}}(\omega) D_{\sigma}\left(z_{0}\right) N_{2, k}\left(\cdot \circ \omega^{-1}, z_{0}\right) d \omega=
$$


(by Lemma 2.17)

$$
=\sum_{\varrho \in I_{\sigma}} \frac{C_{\varrho}}{\left(\bar{u}_{x}\left(z_{0}\right)\right)^{h_{\varrho}}} \prod_{\mu \in J_{\varrho}} D_{\mu} \bar{u}\left(z_{0}\right) \int \Gamma_{z_{0}}(\omega) D_{\varrho}^{\left(z_{0}\right)}\left(z_{0}\right) N_{2, k}\left(\cdot \circ \omega^{-1}, z_{0}\right) d \omega=
$$

(by Proposition 2.23)

$$
=\sum_{\varrho \in I_{\sigma}} \frac{C_{\varrho}}{\left(\bar{u}_{x}\left(z_{0}\right)\right)^{h_{\varrho}}} \prod_{\mu \in J_{\varrho}} D_{\mu} \bar{u}\left(z_{0}\right) \sum_{\nu \in J^{\prime}} \int \Gamma_{z_{0}}(\omega) p_{\nu}(\omega) D_{\nu}^{\left(z_{0}\right)}\left(z_{0}\right) N_{2, k}\left(\cdot \circ \omega^{-1}, z_{0}\right) d \omega .
$$

Each term of this sum is of the form $\int \Gamma_{z_{0}}\left(z_{0}, \zeta\right) \psi\left(\zeta, z_{0}\right) d \zeta$, where $\psi\left(\cdot, z_{0}\right) \in$ $C_{0}^{\infty}(\Omega)$ and, by property (ii) of $N_{2, k}$,

$$
\left|\psi\left(\zeta, z_{0}\right)-\psi(\zeta, z)\right| \leq C d_{z_{0}}\left(z_{0}, z\right)^{\alpha} .
$$

Thus each term can be treated separately as $I_{3, k}^{\sigma}$.

The proof of (ii) is analogous.

Proof of Theorem 1.2 As a consequence of Proposition 3.9 and Lemma 3.10 , the assertion is true for $2 \leq k \leq 6$. Now we assume $k \geq 7$ and we proceed by induction. Let us assume that $u, \bar{u} \in C_{\bar{u}}^{k-1, \alpha}(\Omega)$ and $f=L_{\bar{u}} u \in$ $C_{\bar{u}}^{k-2, \alpha}(\Omega)$. We prove that $u \in C_{\bar{u}}^{k, \alpha}(\Omega)$.

By assumption $u_{y} \in C_{\bar{u}}^{k-4, \alpha}(\Omega)$. Thus, differentiating the equation with respect to the variable $y$, we get $L_{\bar{u}}\left(u_{y}\right)=f_{y}-\bar{u}_{y} u_{y} \in C_{\bar{u}}^{k-5, \alpha}(\Omega)$. By induction, $u_{y} \in C_{\bar{u}}^{k-3, \alpha^{\prime}}(\Omega)$ for $\alpha^{\prime} \in(0, \alpha)$.

On the other hand, $u_{x} \in C_{\bar{u}}^{k-2, \alpha}(\Omega)$ and, differentiating the equation with respect to $x$, we have $L_{\bar{u}}\left(u_{x}\right)=f_{x}-\bar{u}_{x} u_{y} \in C_{\bar{u}}^{k-3, \alpha^{\prime}}(\Omega)$, for $\alpha^{\prime} \in(0, \alpha)$. Therefore, by induction, $u_{x} \in C_{\bar{u}}^{k-1, \alpha^{\prime}}(\Omega)$ for $\alpha^{\prime} \in(0, \alpha)$.

Finally, $Y_{\bar{u}} u \in C_{\bar{u}}^{k-3, \alpha}(\Omega)$ and, differentiating the equation with respect to $Y_{\bar{u}}$, we have $L_{\bar{u}}\left(Y_{\bar{u}} u\right)=Y_{\bar{u}} f+\bar{u}_{x x} u_{y}+2 \bar{u}_{x} u_{x y} \in C_{\bar{u}}^{k-4, \alpha^{\prime}}(\Omega)$, for every $\alpha^{\prime}<\alpha$. Thus, by induction, $Y_{\bar{u}} u \in C_{\bar{u}}^{k-2, \alpha^{\prime}}(\Omega)$ for $\alpha^{\prime} \in(0, \alpha)$. This proves that $u \in C_{\bar{u}}^{k, \alpha}(\Omega)$ for any $\alpha \in(0,1)$.

3.3. The nonlinear operator. In this subsection we prove the regularity of the classical solutions of (1.1).

Proof of Theorem 1.1. We first show that $u \in C_{u}^{1, \alpha}(\Omega)$, for every $\alpha \in$ $(0,1)$. Then, by Theorem 1.2 , it will follow that $u \in C_{u}^{k, \alpha}(\Omega)$ for every $k \in \mathbb{N}$ and $\alpha \in(0,1)$. Thus, by Remark 2.13 , the thesis will be proved.

Since $u$ is a classical solution of (1.1), we have only to show that $X u \in$ $C_{u}^{\alpha}(K)$ for a fixed compact set $K$ and for every $\alpha \in(0,1)$. Representing $u$ 
by formula (3.8), we infer that

$$
X u(z)=\int_{\Omega} X(z) \Gamma_{z_{0}}(\cdot, \zeta) \psi(\zeta) d \zeta
$$

for some suitable $\psi \in C_{0}(\Omega)$ and $z_{0} \in \Omega$. We have

$$
\left|X u(z)-X u\left(z_{0}\right)\right| \leq A_{1}\left(z, z_{0}\right)+A_{2}\left(z, z_{0}\right),
$$

where, for $\Omega_{M}$ defined in (2.33),

$$
\begin{aligned}
& A_{1}\left(z, z_{0}\right)=\int_{\Omega \backslash \Omega_{M}}\left|\left(X(z)-X\left(z_{0}\right)\right) \Gamma_{z_{0}}(\cdot, \zeta)\right||\psi(\zeta)| d \zeta \\
& A_{2}\left(z, z_{0}\right)=\int_{\Omega_{M}}\left|\left(X(z)-X\left(z_{0}\right)\right) \Gamma_{z_{0}}(\cdot, \zeta)\right||\psi(\zeta)| d \zeta
\end{aligned}
$$

By Remark 2.19, we obtain the following estimate of $A_{1}\left(z, z_{0}\right)$ :

$$
A_{1}\left(z, z_{0}\right) \leq C_{1} \int_{\Omega \backslash \Omega_{M}}\left(d_{z_{0}}(z, \zeta)^{-Q+1}+d_{z_{0}}\left(z_{0}, \zeta\right)^{-Q+1}\right) d \zeta \leq C d_{z_{0}}\left(z_{0}, z\right) .
$$

On the other hand, by Proposition 2.20, we have

$$
\begin{aligned}
A_{2}\left(z, z_{0}\right) & \leq C \int_{\Omega_{M}}\left(d_{z_{0}}\left(z_{0}, z\right) d_{z_{0}}\left(z_{0}, \zeta\right)^{-Q}+d_{z_{0}}\left(z_{0}, z\right)^{\alpha} d_{z_{0}}\left(z_{0}, \zeta\right)^{-Q+1}\right) d \zeta \\
& \leq C d_{z_{0}}\left(z_{0}, z\right)^{\alpha}
\end{aligned}
$$

for every $\alpha \in(0,1)$. Thus the proof is completed.

Acknowledgments. We thank E. Barucci for proposing to us the problem and for many interesting discussions on its financial aspects.

\section{REFERENCES}

[1] F. Antonelli, E. Barucci, and M.E. Mancino, Backward-forward stochastic differential utility: Existence, optimal consumption and equilibrium analysis, to appear.

[2] M. Bramanti and L. Brandolini, $L^{p}$ estimates for uniformly hypoelliptic operators with discontinuous coefficients on homogeneous groups, to appear.

[3] M. Bramanti, Cerutti, and M. Manfredini, $L^{p}$ estimates for some ultraparabolic operators with discontinuous coefficients, J. Math. Anal. Appl., 200 (1996), 332-354.

[4] L. Capogna, D. Danielli, and N. Garofalo, An embedding theorem and the Harnack inequality for nonlinear subelliptic equations, Comm. Partial Diff. Eq., 18 (1993), 1765-1794.

[5] F. Chiarenza, M. Frasca, and P. Longo, Interior $W^{2, p}$ estimates for non-divergence elliptic equations with discontinuous coefficients, Ric. Mat., 40 (1991), 149-168.

[6] G. Citti, $C^{\infty}$ regularity of solutions of a quasilinear equation related to the Levi operator, Ann. Scuola Norm. Sup. Pisa Cl. Sci., Serie IV, Vol. XXIII (1996), 483-529.

[7] G. Citti and A. Montanari, $C^{\infty}$ regularity of solutions of an equation of Levi's type in $R^{2 n+1}$, Ann. Mat. Pura Appl., 4, to appear. 
[8] D. Duffie and L. Epstein, Stochastic differential utility, Econometrica, 60 (1992), 353394.

[9] L. Epstein and S. Zin, Substitution, risk aversion, and the temporal behavior of consumption and asset returns: a theoretical framework, Econometrica, 57 (1989), 937969.

[10] M. Escobedo, J.L. Vazquez, and E. Zuazua, Entropy solutions for diffusion-convection equations with partial diffusivity, Trans. Am. Math. Soc., 343 (1994), 829-842.

[11] G.B. Folland, Subelliptic estimates and function spaces on nilpotent Lie groups, Ark. Mat., 13 (1975), 161-207.

[12] B. Franchi and E. Lanconelli, Hölder regularity theorem for a class of linear nonuniformly elliptic operators with measurable coefficients, Ann. Sc. Norm. Super. Pisa, Cl. Sci., IV. Ser., 10 (1983), 523-541.

[13] G.B. Folland and E.M. Stein, Estimates for the $\bar{\partial}_{b}$ complex and analysis on the Heisenberg group, Comm. Pure Appl. Math., 20 (1974), 429-522.

[14] L. Hörmander, Hypoelliptic second order differential equations, Acta Math., 119 (1967), 147-171.

[15] G. Lu, Weighted Poincaré and Sobolev inequalities for vector fields satisfying Hörmander's condition and applications, Rev. Math Iberoamericana, 8 (1992), 367439.

[16] A. Lunardi, Schauder estimates for a class of degenerate elliptic and parabolic operators with unbounded coefficients in $\mathbb{R}^{n}$, Ann. Scuola Norm. Sup. Pisa, Serie IV, Vol. XXIV (1997), 133-164.

[17] A. Nagel, E.M. Stein, and S. Wainger, Balls and metrics defined by vector fields I: basic properties, Acta Math., 155 (1985), 103-147.

[18] R. Peszek, PDE models for pricing stocks and options with memory feedback, Appl. Math. Finance, 2 (1995), 211-223.

[19] S. Polidoro, On a class of ultraparabolic operators of Kolmogorov-Fokker-Planck type, Le Matematiche, 49 (1994), 53-105.

[20] L.P. Rothschild and E.M. Stein, Hypoelliptic differential operators on nilpotent groups, Acta Math., 137 (1977), 247-320.

[21] A. Sánchez-Calle, Fundamental solutions and geometry of the sum of squares of vector fields, Invent. Math., 78 (1984), 143-160.

[22] A.N. Shiryayev (ed.), "Selected works of A. N. Kolmogorov," vol. II, Probability theory and mathematical statistics, Kluver Academic Publishers, Dodrecht-BostonLondon (1991).

[23] A.I. Vol'pert and S.I. Hudjaev, Cauchy's problem for degenerate second order quasilinear parabolic equations, Math. USSR Sb., 7 (1969), 365-387.

[24] S.K. Vodop'yanov and I.G. Markina, Exceptional sets for solutions to subelliptic equations, Siberian Math. J., 36 (1995), 694-706.

[25] C. Xu, Regularity for quasilinear second-order subelliptic equations, Comm. Pure Appl. Math., 45 (1992), 77-96.

[26] C. Xu, Dirichlet problems for the quasilinear second order subelliptic equations, Acta Math. Sinica, New Ser., 12 (1996), 18-32. 